\title{
Data report: paleomagnetic and rock magnetic measurements on Hole U1301B basaltic samples ${ }^{1}$
}

\author{
William W. Sager, ${ }^{2}$ Bernard A. Housen, ${ }^{3}$ and Lisa M. Linville ${ }^{3}$
}

\section{Chapter contents}

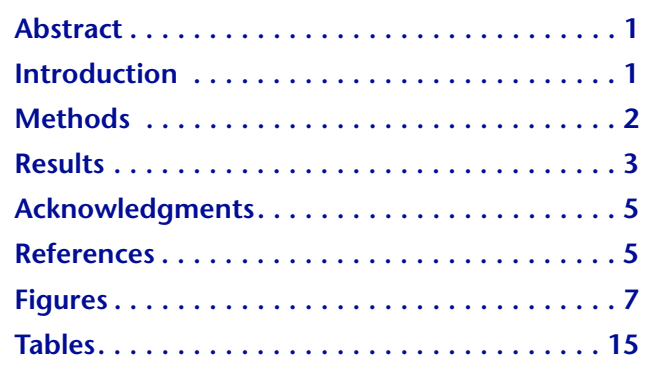

'Sager, W.W., Housen, B.A., and Linville, L.M., 2008. Data report: paleomagnetic and rock magnetic measurements on Hole U1301B basaltic samples. In Fisher, A.T., Urabe, T., Klaus, A., and the Expedition 301 Scientists, Proc. IODP, 301: College Station, TX (Integrated Ocean Drilling Program Management International, Inc.). doi:10.2204/iodp.proc.301.204.2008 2Department of Oceanography, Texas A\&M University, College Station TX 77843, USA. wsager@tamu.edu

${ }^{3}$ Geology Department, Western Washington University, Bellingham WA 98225, USA.

\section{Abstract}

During Integrated Ocean Drilling Program Expedition 301, 235 m of upper igneous crust was cored in Hole U1301B, in an area of hydrothermal circulation on Juan de Fuca Ridge, providing an opportunity to better understand ocean crust magnetization. We studied the paleomagnetism of this section using a variety of techniques. Natural remanent magnetization (NRM) and alternating-field or thermal demagnetization measurements were made on 330 individual samples of igneous rock from both the working-half and archive-half cores. Rock magnetic characterization measurements were made on a subset of samples to better understand the source of the magnetization. These measurements included hysteresis parameters, Curie temperature, and isothermal remanent magnetization (IRM) acquisition. In addition, thin sections were examined for several samples in an effort to visually identify magnetic grains. Igneous samples from Hole U1301B display a range of NRM values between 1.3 and $73.0 \mathrm{~A} / \mathrm{m}$ with a median value of $6.9 \mathrm{~A} / \mathrm{m}$, which are values typical of ocean crust basalts. IRM acquisition curves are consistent with titanomagnetite as the magnetic remanence carrier. Hysteresis parameters indicate single domain and pseudosingle domain behavior, despite large grain sizes seen in scanning electron microphotographs. The apparent paradox is resolved by observation of grain textures that indicate that large grains are split into small domains. Magnetization direction characteristics vary and have been divided into five types by demagnetization patterns. The many patterns suggest that the remanent magnetization of some samples has been altered by secondary processes, such as hydrothermal metamorphism.

\section{Introduction}

During Integrated Ocean Drilling Program (IODP) Expedition 301, 3.5 Ma age crust was drilled on the flank of the Juan de Fuca Ridge at Site U1301, located at $47^{\circ} 45.2^{\prime} \mathrm{N}, 127^{\circ} 45.8^{\prime} \mathrm{W}$ (see the "Expedition 301 summary" chapter). The site targeted an area of hydrothermal fluid flow for an experiment examining the hydrology of the ocean crust. Although the ridge flank is buried by unusually thick sediments because of the proximity of land, it is otherwise the site of normal oceanic crust. More than $200 \mathrm{~m}$ of upper oceanic crust basalt was cored in Hole U1301B, making it 
one of the relatively few sites where such a large section of crustal rock has been sampled. For scientists who study paleomagnetism, this represents an excellent opportunity to learn more about the magnetization of the upper crust.

We sampled the basalt section from Hole U1301B with the goal of learning about the magnetization properties of the recovered basalt samples. Because the crust appears normal, we expected to find that the basalts were magnetized in the direction of the magnetic field 3.5 m.y. ago, when the crust at Site U1301 was formed. Our working hypothesis was that the samples should behave as typical ocean crust basalts, should be normally polarized (because the site is on a normal polarity magnetic anomaly), and should give magnetization inclinations that are similar to the geocentric axial dipole inclination for the site $\left(65.6^{\circ}\right)$. We also expected that some of the samples would display evidence of hydrothermal alteration, owing to the location of the site in an area of hydrothermal circulation.

\section{Background}

Hole U1301B was one of four holes drilled at Site U1301. The drill string encountered the seafloor at a depth of 2656 meters below sea level (mbsl) and penetrated to 582.8 meters below seafloor (mbsf). Drilling in Hole U1301B showed that the upper $265.2 \mathrm{~m}$ of the section is sedimentary. Thus, the lower 317.6 $\mathrm{m}$ of the drilled section penetrated upper crustal igneous rocks.

Because of problems coring the fractured upper part of the igneous section at nearby Site 1026 during Ocean Drilling Program (ODP) Leg 168 (Shipboard Scientific Party, 1997), the upper $85 \mathrm{~m}$ of the igneous section in Hole U1301B was not cored. Only 5\% recovery was obtained throughout this section during Leg 168, so the upper igneous section is not well known. After casing for hole stability during Expedition 301, igneous rocks below $85 \mathrm{~m}$ beneath the sediment/basalt contact were continuously cored. A total of $69.1 \mathrm{~m}$ of igneous core was recovered from $235 \mathrm{~m}$ of cored section for a recovery percentage of $\sim 30 \%$.

The cored igneous section was divided into eight units defined by changes in lava morphology, rock texture, and grain size (see the "Site U1301" chapter). Units $1,3,5,7$, and 8 are characterized by pillow basalts, whereas Units 2, 4, and 6 are massive units (Fig. F20 in the "Site U1301" chapter). The massive units are generally thin $(<10 \mathrm{~m}$ thick), so most of the igneous section (and samples recovered) consists of pillow basalts.
Pillows from the cores are generally sparsely vesicular and micro- or cryptocrystalline in texture. Massive basalts are similar but have few vesicles. Both types of rock are normal depleted mid-ocean-ridge basalts (MORB) as indicated by geochemical analyses (see the "Site U1301" chapter). Alteration in the core samples ranges from slight to moderate $(5 \%-$ $25 \%)$, with the slightly altered rocks generally dark gray in appearance. The most unaltered parts of the samples are in flow interiors, and the alteration has proceeded to saponite grade (see the "Site U1301" chapter). More intense alteration has proceeded generally along cracks and veins, often forming alteration halos around such features. The halos have varied colors (black, brown, and green) depending on the mix of secondary minerals. Potentially magnetic iron oxyhydroxides were found in $\sim 44 \%$ of the veins examined, pyrite was found in $\sim 3 \%$, and goethite was found in one vein.

\section{Methods}

\section{Shipboard measurements}

During Expedition 301, the igneous section was extensively sampled for paleomagnetic study, with measurements made from a total of 330 discrete samples (Tables T1, T2). All measurements were made on discrete samples because the igneous core was too discontinuous to allow for reliable measurements from whole core sections. A total of 172 discrete $\sim 15 \mathrm{~cm}^{3}$ paleomagnetic samples were taken at an average spacing of $\sim 2 / \mathrm{m}$ of recovered core. These samples were acquired using a two-blade saw to produce cubes $\sim 25 \mathrm{~mm}$ on a side. In order to obtain a greater number of paleomagnetic data points, three other types of samples were also measured. One was $\sim 15 \mathrm{~cm}^{3}$ discrete cube samples, similar to the paleomagnetic samples except that they were taken for physical property measurements (23 samples). Another was irregular-sized intact oriented pieces $(\sim 50$ $200 \mathrm{~cm}^{3}$ ) of the archive-half core (127 samples). In addition, eight pairs of small irregular-sided pieces, usually $<5 \mathrm{~cm}^{3}$ in volume, were collected to investigate the differences in magnetic properties inside and outside of halo alteration zones.

All but the small irregular samples were measured on the shipboard 2G Enterprises model 760R passthrough cryogenic superconducting quantum interference device (SQUID) magnetometer (see the "Methods" chapter). Measurements were made using the usual ODP core reference frame with the $\mathrm{x}$-axis in the upcore direction (see the "Methods" chapter). The characteristic remanent magnetization (ChRM) 
was isolated using either stepwise alternating-field (AF) or thermal demagnetization. All AF demagnetization steps were run on the $2 \mathrm{G}$ Enterprises model 2G600 inline demagnetizer that is a part of the passthrough magnetometer system. Thermal heating was accomplished with a Schoenstedt model TSD-1 magnetically shielded oven.

Because the measurement of physical property samples and archive-half core pieces had to be nondestructive, AF demagnetization was used on these samples. Typically, AF demagnetization was done in $5 \mathrm{mT}$ steps from 10 to $40 \mathrm{mT}$ and in $10 \mathrm{mT}$ steps for higher fields. In contrast, most of the samples acquired for paleomagnetic study were thermally demagnetized (see Table T1). Most thermal demagnetization analyses used $50^{\circ} \mathrm{C}$ steps from $150^{\circ}$ to $550^{\circ} \mathrm{C}$; however, other temperatures were used in some instances to explore magnetization properties.

$\mathrm{AF}$ and thermal demagnetization data were plotted for each sample on an orthogonal vector diagram (Zijderfeld, 1967) to aid in interpreting magnetization components and directions. ChRM is assumed to be shown by a section of high-temperature or high-AF field univectorial decay observed on the orthogonal vector plot. Magnetization directions were calculated using principal component analysis (Kirschvink, 1980) from that section of univectorial decay. With only a few exceptions, all of the magnetization directions were determined from calculations that were not anchored to the orthogonal vector plot origin. Typically four to six measurements were used for the principal component analysis. For two samples, the principal component analysis was constrained by only two measurements, so these calculations were anchored to the origin.

\section{Shore-based measurements}

Shore-based measurements were made in the paleomagnetic laboratory at Western Washington University with the intent of better understanding the source and characteristics of the magnetization in Hole U1301B basalts. Measurements of Curie temperature and magnetic hysteresis parameters were made on 32 discrete $\sim 15 \mathrm{~cm}^{3}$ cube samples (Table T2). Magnetization direction studies were carried out on these and four additional cube samples. In addition, magnetic hysteresis and isothermal remanent magnetization (IRM) acquisition measurements were conducted on eight pairs of samples in which the two samples of each pair come from an alteration halo and the adjacent slightly altered rock (Tables T3, T4).

All samples were treated to detailed AF or thermal demagnetization routines, with all but 4 of the 36 samples being thermally demagnetized. AF demag- netization was carried out in a D-Tech D2000 AF demagnetizer. Compared with previous shipboard analysis, demagnetization steps were more closely spaced at low field values (2-3 $\mathrm{mT}$ steps to $10 \mathrm{mT}$ ), the same $5 \mathrm{mT}$ steps were used from 10 to $100 \mathrm{mT}$, and $20 \mathrm{mT}$ steps were used to $200 \mathrm{mT}$. Thermal demagnetization was also generally more detailed than shipboard analyses, with steps of $10^{\circ}-20^{\circ} \mathrm{C}$ being commonly used. Thermal demagnetization was carried out to $450^{\circ}-580^{\circ} \mathrm{C}$, depending on results from any particular sample. Heating was done in an argon-filled environment to prevent oxidation from occurring at high temperatures. Magnetization direction analysis for each of these samples was carried out in the same manner as for the shipboard samples.

Hysteresis loops were measured using a MicroMag vibrating sample magnetometer (VSM) at room temperature in fields of up to $10 \mathrm{kOe}$. Hysteresis loops before and after heating were also taken for samples for which Curie temperatures were measured in the presence of a magnetic field. Paramagnetic contributions to the hysteresis curves were minimized in most cases by a slope correction using MicroMag software. IRM and backfield curves were also determined with the VSM.

To determine Curie temperature, magnetic susceptibility was measured as a function of temperature using a Kappabridge magnetic susceptibility meter with a furnace attachment. Chips from each sample were powdered and cycled from room temperature to $700^{\circ} \mathrm{C}$ and back to $40^{\circ} \mathrm{C}$ in the presence of argon gas to minimize oxidation. Chips from two representative samples were then cut and powdered for closer examination. These samples were run in the same manner as previously described but to a maximum temperature of $320^{\circ} \mathrm{C}$. Curie temperatures were determined by the intersecting tangents method from graphs of magnetic susceptibility versus temperature. To gauge the accuracy of susceptibility-based measurements, four paired samples were selected for direct Curie temperature measurement with a furnace-equipped VSM. Curie temperatures for these samples were determined by the second derivative method outlined by Tauxe (1998). Thin sections from these sample pairs were also made, polished, and coated in carbon for scanning electron microscope (SEM) examination.

\section{Results}

\section{NRM and directional measurements}

Sample natural remanent magnetization (NRM) and directional measurements are given in Table T1. NRM values range from 1.3 to $73.0 \mathrm{~A} / \mathrm{m}$ with a me- 
dian value of $6.9 \mathrm{~A} / \mathrm{m}$. Most samples have NRM values $<10 \mathrm{~A} / \mathrm{m}$, and only seven have NRM values $>50$ $\mathrm{A} / \mathrm{m}$ (Fig. F1). These values are typical of submarine basalts, and the skewed distribution with a small number of large NRM values is also commonly observed (Johnson and Pariso, 1993; Johnson et al., 1996; Zhao et al., 2006). High NRM values are scattered more or less evenly throughout the section (Fig. F1).

During demagnetization, Hole U1301B samples show a remarkable variety of behavior and ChRM directions (Figs. F2, F3). Samples gave better results with thermal demagnetization, which is why we used that technique for most of the demagnetization experiments. Some AF-demagnetized samples displayed magnetizations that veered from the origin at high field values (Fig. F2D), implying some sort of spurious magnetization imparted by the demagnetization equipment (e.g., a rotational remanent magnetization or gyroremanent magnetization). Most samples displayed a large, steeply downward NRM direction that is probably indicative of the drillstring overprint that is common among paleomagnetic samples cored onboard the JOIDES Resolution (see Fig. F2A, F2C, F2E) (Acton et al., 2002; Fuller et al., 2006). In some low-coercivity samples, this overprint dominated magnetizations to the point that it was difficult to determine the ChRM. In samples from the upper part of the igneous section, both $\mathrm{AF}$ and thermal demagnetization revealed a ChRM with a downward-directed (positive) inclination between $\sim 30^{\circ}$ and $90^{\circ}$ (Figs. F2A, F2C, F2F, F3). This magnetization direction indicates normal polarity in the Northern Hemisphere. Some samples give a reversed polarity inclination (i.e., negative or upward). Typically, this ChRM is found at high-temperature steps and is masked by a drill string overprint or a lower temperature normal component similar in dip to the normal ChRM samples (Fig. F2E). A few samples, such as 301-U1301B-6R-2, 35 cm (Fig. F2B), are reversed with no evidence of a downward overprint, and such samples may have been inverted during handling.

To examine ChRM trends, we arbitrarily classify ChRM directions into five classes:

- Type 1 has low inclination, reversed ChRM.

- Types 2 and 3 have normal ChRM inclinations, with those of type 2 having values below $\sim 40^{\circ}$ and those of Type 3 having higher values.

- Type 4 is characterized by a dominant drill string overprint (typically low-coercivity samples).

- Type 5 is has two components, a high-temperature reversed inclination and a lower temperature normal direction.
Most samples are Types 2 or 3 (Table T1), especially in the upper part of the section. In the lower part of the section, below 470 mbsf, Types 2 and 3 are still common, but all of the different types are represented with little apparent correlation between adjacent or nearby samples. This part of the section also displays ChRM inclinations with highly scattered values, including negative (apparently reversed) inclinations (Fig. F3).

Because Site U1301 is on a normal polarity magnetic anomaly, we have interpreted the normal inclinations as "normal" and the reversed inclinations as spurious (see the "Site U1301" chapter). Even in the apparently normal-behaving upper section, normal polarity inclinations do not precisely match the expected inclination. The average inclination for the section above $470 \mathrm{mbsf}$ is $53.5^{\circ}$, which is less than the geocentric axial dipole inclination for the site of $66.5^{\circ}$. Even if this value is corrected for the slight shallowing caused by averaging azimuthally unoriented samples (corrected expected inclination = $64.2^{\circ}$ using Cox and Gordon, 1984) the difference is $>10^{\circ}$.

\section{Rock magnetic measurements}

IRM acquisition curves all saturate quickly in low (<100 mT) applied fields (Fig. F4; Table T2). This lowfield IRM saturation is characteristic of titanomagnetite grains. The flat high-field sections of the curves indicate the absence of high-coercivity magnetic minerals, such as hematite. Observed differences between samples from alteration halos and nearby samples from slightly altered core are small but systematic. Samples from alteration halos consistently require slightly greater applied fields to reach saturation (Fig. F4), implying that the alteration slightly raises the coercivity of the bulk assemblage of magnetic grains.

Thermomagnetic curves for Hole U1301B basalt samples are usually nonreversible, although some samples do give reversible curves (Fig. F5). Nonreversibility implies changes in the magnetic mineral assemblage caused by heating. In these samples, we are probably seeing the conversion of maghemite into other magnetic minerals. The thermomagnetic curves give Curie temperatures for Hole U1301B samples ranging from $128^{\circ}$ to $370^{\circ} \mathrm{C}$ (Tables T3, T4) with a median value of $339^{\circ} \mathrm{C}$. Most samples give values in the $240^{\circ}-320^{\circ} \mathrm{C}$ range. These values are typical for altered ocean basalts with titanomagnetite magnetic grains.

Magnetic hysteresis data (Tables T3, T4) show that all samples have saturation remanence/saturation magnetization $\left(M_{\mathrm{r}} / M_{\mathrm{s}}\right)$ ratios $<0.5$ and remanent co- 
ercive force/ordinary coercive force $\left(H_{\mathrm{cr}} / H_{\mathrm{c}}\right)$ ratios of $<5$. Most samples plot near the single domain (SD) field on the Day plot (Fig. F6), although some samples have hysteresis parameter ratios that fall in the pseudosingle domain (PSD) region. Of the paired samples from alteration halos and nearby slightly altered rock, it appears that the more altered samples plot preferentially toward the SD region of the plot, suggesting that the alteration process makes magnetic grains behave more like SD grains. Most samples plot near model lines (Dunlop, 2002) for mixtures of SD and multidomain (MD) grains. Given microscopic observations (see below) that indicate both large and small magnetic grains, these samples may indeed be displaying the behavior of a SD/MD grain mixture.

On a Day plot, samples with different demagnetization behaviors show no indication of clustering (Fig. F7). Samples with each different demagnetization behavior are found scattered throughout the hysteresis ratio range of the entire sample population. This implies that different types of demagnetization behavior are not a function of magnetic grain size.

SEM photomicrographs (Fig. F8) show that large titanomagnetite grains are found in sample thin sections. These grains are often rectangular or deltoid in appearance, which is typical of titanomagnetite. Many of these grains are tens of micrometers in diameter, which is far larger than the normal size for SD or PSD grains. Indeed, grains of this size should behave as multidomain grains. An explanation for the difference is that many of the grains show trellis, dendritic, skeletal, and cruciform patterns within the grains, indicating that the grains are broken up internally into smaller pieces. Furthermore, the surfaces of these grains are often invaded by cracks, implying further divisions. In sum, the naturally occurring fractures and crystal structures tend to be much smaller than the whole grain. In addition, electron dispersive scattering (EDS) tests imply that titanomagnetites are also found within the groundmass, so there is likely to be a very small size grain component also existing within these samples.

\section{Acknowledgments}

This research used samples and data provided by the Integrated Ocean Drilling Program (IODP). IODP is sponsored by the U.S. National Science Foundation (NSF) and participating countries under management of Joint Oceanographic Institutions (JOI), Inc. (renamed The Consortium for Ocean Leadership). W. Sager was supported for this research by a grant from the JOI/U.S. Science Support Program. L. Linville was supported with a supplement to that grant for undergraduate education. B. Housen acknowledges support from NSF grants EAR-MRI-0421457 for the vibrating sample magnetometer and EAR-IR9727032 for the cryogenic magnetometer in the laboratory at Western Washington University.

\section{References}

Acton, G.D., Okada, M., Clement, B.M., Lund, S.P., and Williams, T., 2002. Paleomagnetic overprints in ocean sediment cores and their relationship to shear deformation caused by piston coring. J. Geophys. Res., 107(B4):2067. doi:10.1029/2001JB000518

Cox, A., and Gordon, R.G., 1984. Paleolatitudes determined from paleomagnetic data from vertical cores. Rev. Geophys. Space Phys., 22(1):47-71. doi:10.1029/ RG022i001p00047

Dunlop, D.J., 2002. Theory and application of the Day plot $\left(M_{\mathrm{rs}} / M_{\mathrm{s}}\right.$ versus $\left.H_{\mathrm{cr}} / H_{\mathrm{c}}\right), 1$. Theoretical curves and tests using titanomagnetite data. J. Geophys. Res., 107(B3):2056. doi:10.1029/2001JB000486

Fuller, M., Molina-Garza, R., Touchard, Y., and Kidane, T., 2006. Paleomagnetic records from carbonate legs in the Southern Oceans and attendant drilling and coring related effects. In Sager, W.W., Acton, G.D., Clement, B.M., and Fuller, M. (Eds.), ODP Contributions to Paleomagnetism. Phys. Earth Planet. Int., 156(3-4):242-260. doi:10.1016/j.pepi.2005.08.007

Johnson, H.P., and Pariso, J.E., 1993. Variations in oceanic crustal magnetization: systematic changes in the last 160 million years. J. Geophys. Res., 98(B1):435-445. doi:10.1029/92JB01322

Johnson, H.P., Van Patten, D.V., and Sager, W.W., 1996. Age-dependent variation in the magnetization of seamounts. J. Geophys. Res., 101(B6):13701-13714. doi:10.1029/96JB00537

Kirschvink, J.L., 1980. The least-squares line and plane and the analysis of palaeomagnetic data. Geophys. J. R. Astron. Soc., 62(3):699-718.

Shipboard Scientific Party, 1997. Rough basement transect (Sites 1026 and 1027). In Davis, E.E., Fisher, A.T., Firth, J.V., et al., Proc. ODP, Init. Repts., 168: College Station, TX (Ocean Drilling Program), 101-160. doi:10.2973/ odp.proc.ir.168.105.1997

Tauxe, L., 1998. Paleomagnetic Principles and Practice: Dordrecht, Netherlands (Kluwer Academic Publishers.

Zhao, X., Riisager, P., Antretter, M., Carlut, J., Lippert, P., Liu, Q., Galbrun, B., Hall, S., Delius, H., and Kanamatsu, T., 2006. Unraveling the magnetic carriers of igneous cores from the Atlantic, Pacific, and the southern Indian oceans with rock magnetic characterization. In Sager, W.W., Acton, G.D., Clement, B.M., and Fuller, M. (Eds.), ODP Contributions to Paleomagnetism. Phys. Earth Planet. Int., 156(3-4):294-328. doi:10.1016/ j.pepi.2005.08.005 
Zijderveld, J.D.A., 1967. AC demagnetization of rocks: analysis of results. In Collinson, D.W., Creer, K.M., and Runcorn, S.K. (Eds.), Methods in Palaeomagnetism: New York (Elsevier), 254-286.
Initial receipt: 29 May 2007

Acceptance: 18 February 2008

Publication: 7 April 2009

MS 301-204 
Figure F1. Natural remanent magnetization (NRM) of basalt samples, Hole U1301B.

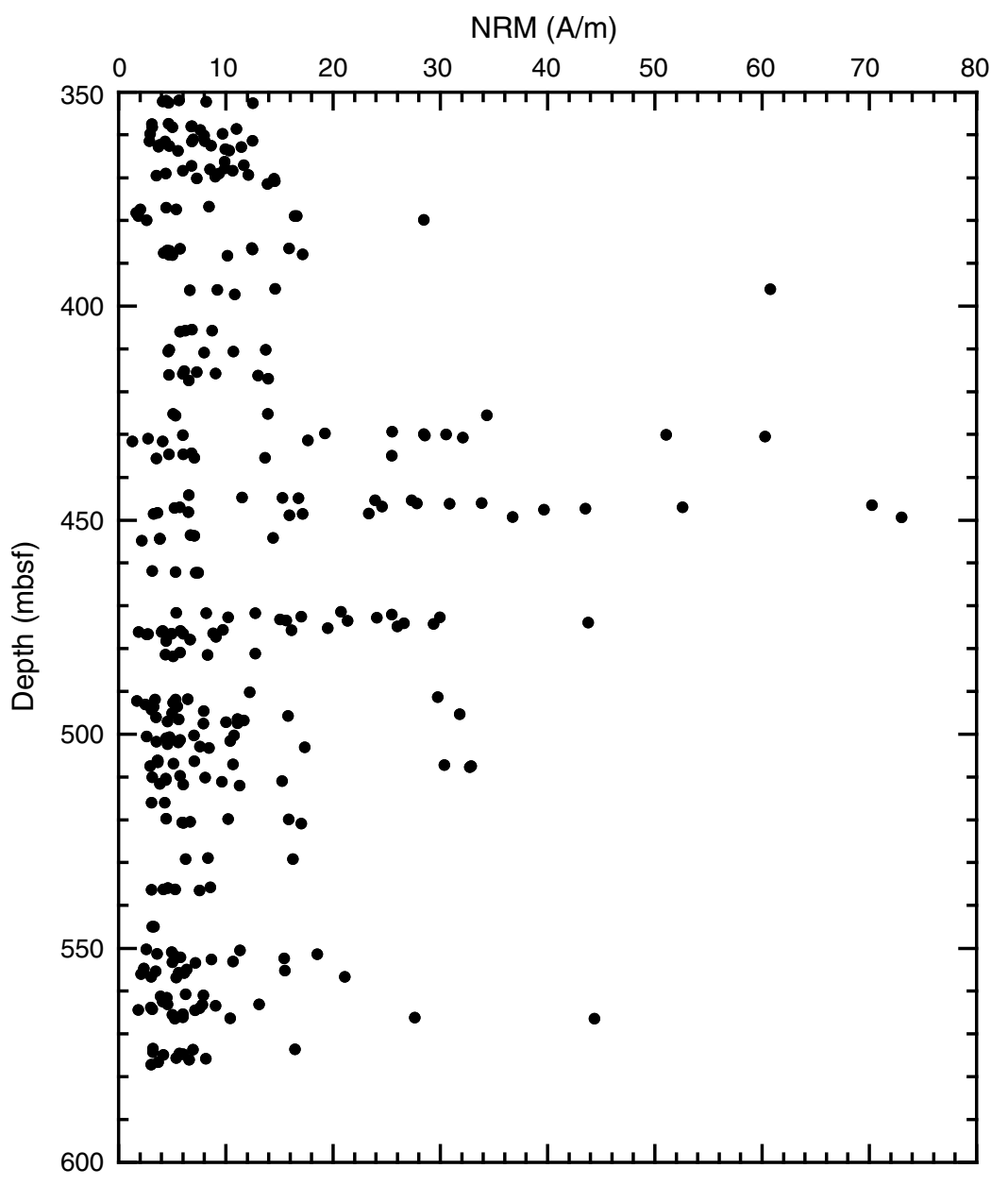


Figure F2. Basalt sample demagnetization behavior, Hole U1301B. A-D. Alternating-field demagnetization. (A) Sample 301-U1301B-30R-1, $98 \mathrm{~cm}$, has a positive inclination, indicating normal polarity at the drill site. (B) Although Sample 301-U1301B-6R-2, $35 \mathrm{~cm}$, would normally be considered to have a reversed magnetization (i.e., negative inclination), the sample gives an isolated result and shows no downward overprint, as do most other samples, so it is thought to have been accidentally inverted during curation. (C) Sample 301U1301B-32R-1, $36 \mathrm{~cm}$, shows a large downward drill string overprint. (D) Sample 301-U1301B-3R-1, $110 \mathrm{~cm}$, veers from the simple univectorial decay at high-field demagnetization steps. E-F. Thermal demagnetization. (E) Sample 301-U1301B-30R-1, $99 \mathrm{~cm}$, shows two components with a high-temperature reversed polarity characteristic magnetization, whereas (F) Sample 301-U1301B-5R-2, $109 \mathrm{~cm}$, shows a normal polarity magnetization after removal of the drill string overprint. The latter is an example of Type 2 or 3 behavior. Sample 301U1301B-30R-1, $99 \mathrm{~cm}$, is an example of Type 5 behavior. If the inclination were shallower, it would be classified as Type 1 (see "NRM and directional measurements" for discussion of behavior classes).

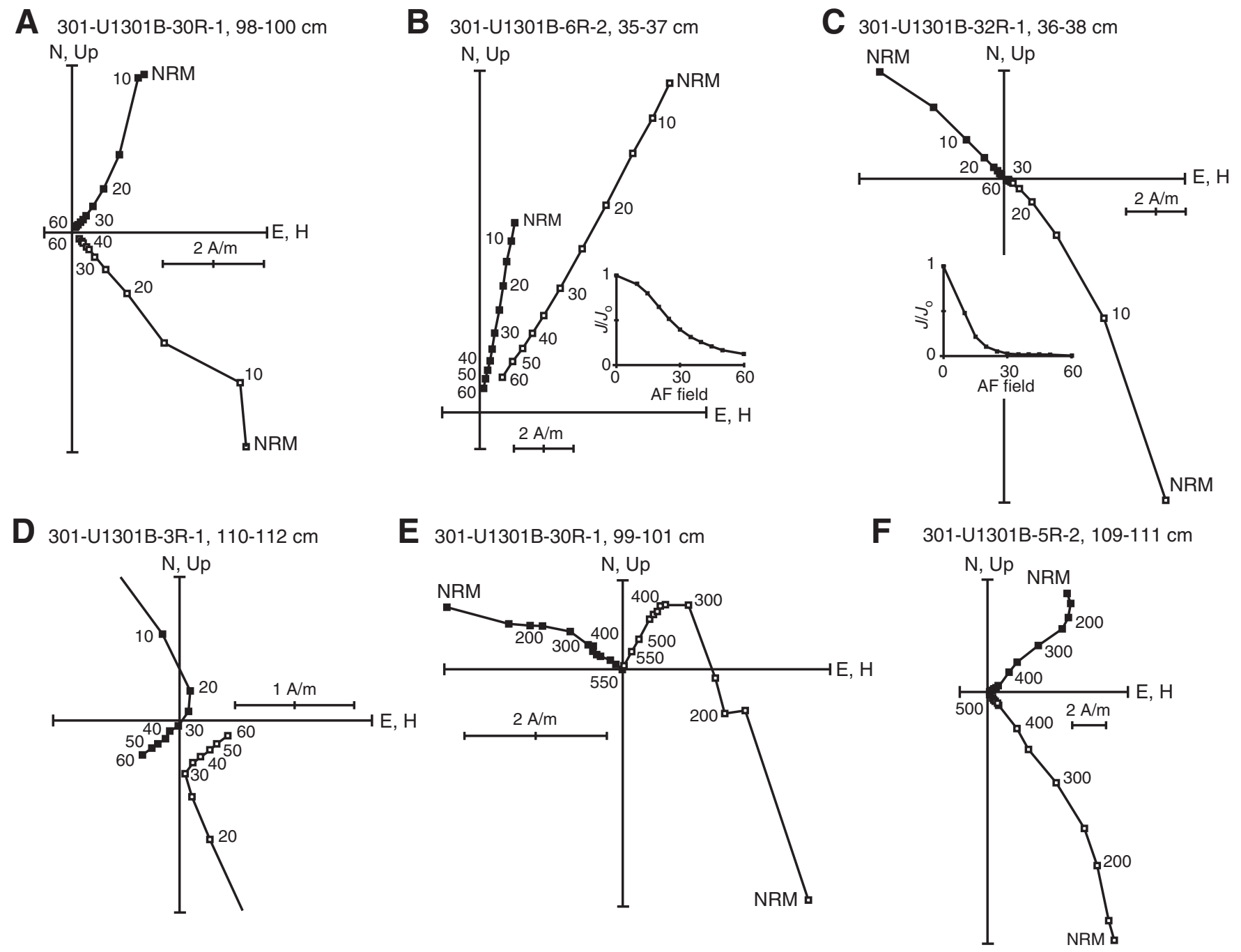


Figure F3. ChRM inclination values for basalt samples, Hole U1301B. Dotted vertical lines show expected geocentric axial dipole inclination for site location.

Inclination $\left({ }^{\circ}\right)$

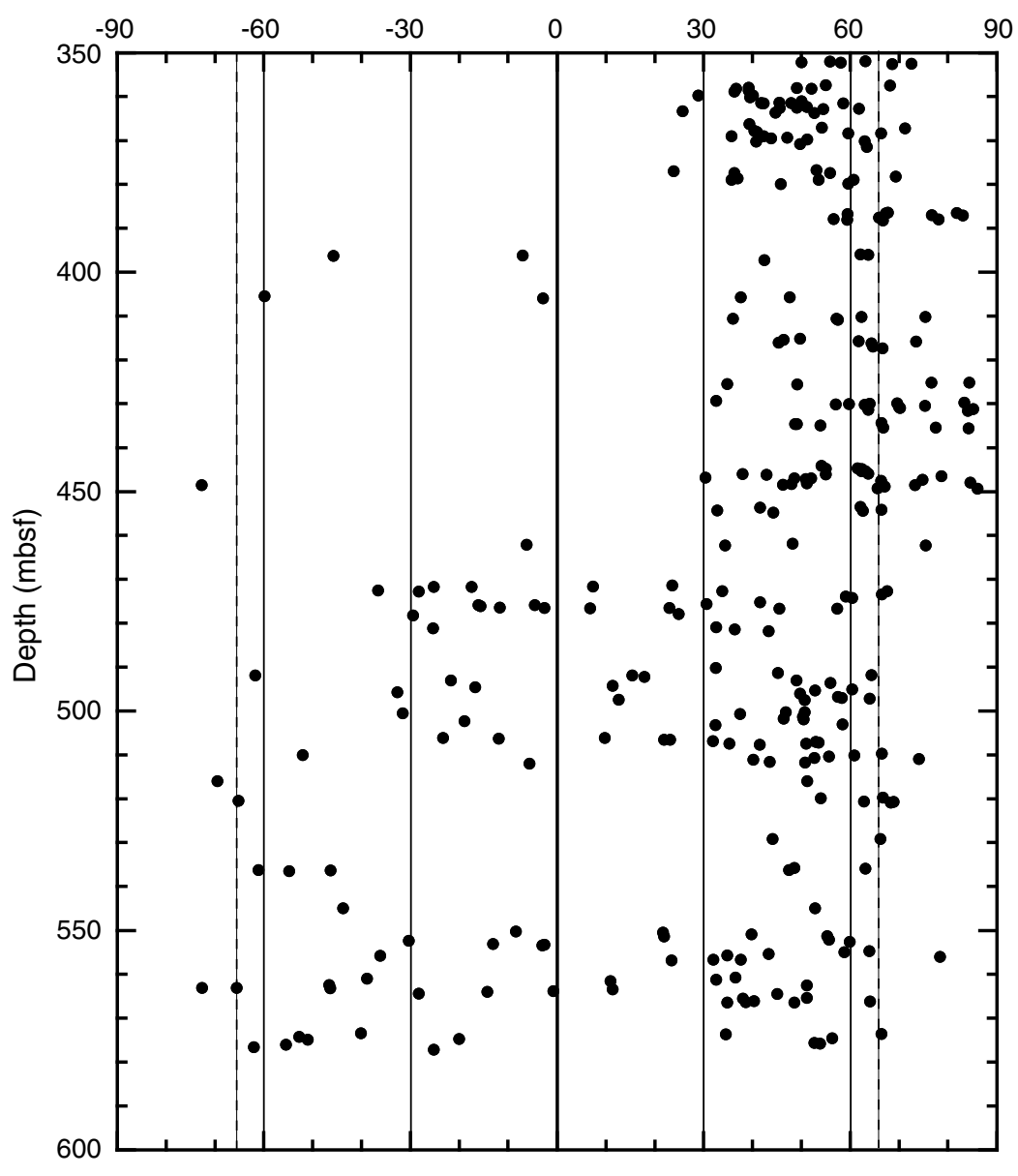


Figure F4. IRM acquisition curve for two representative samples, Hole U1301B. One sample is from relatively unaltered core and the other is from an adjacent alteration halo.

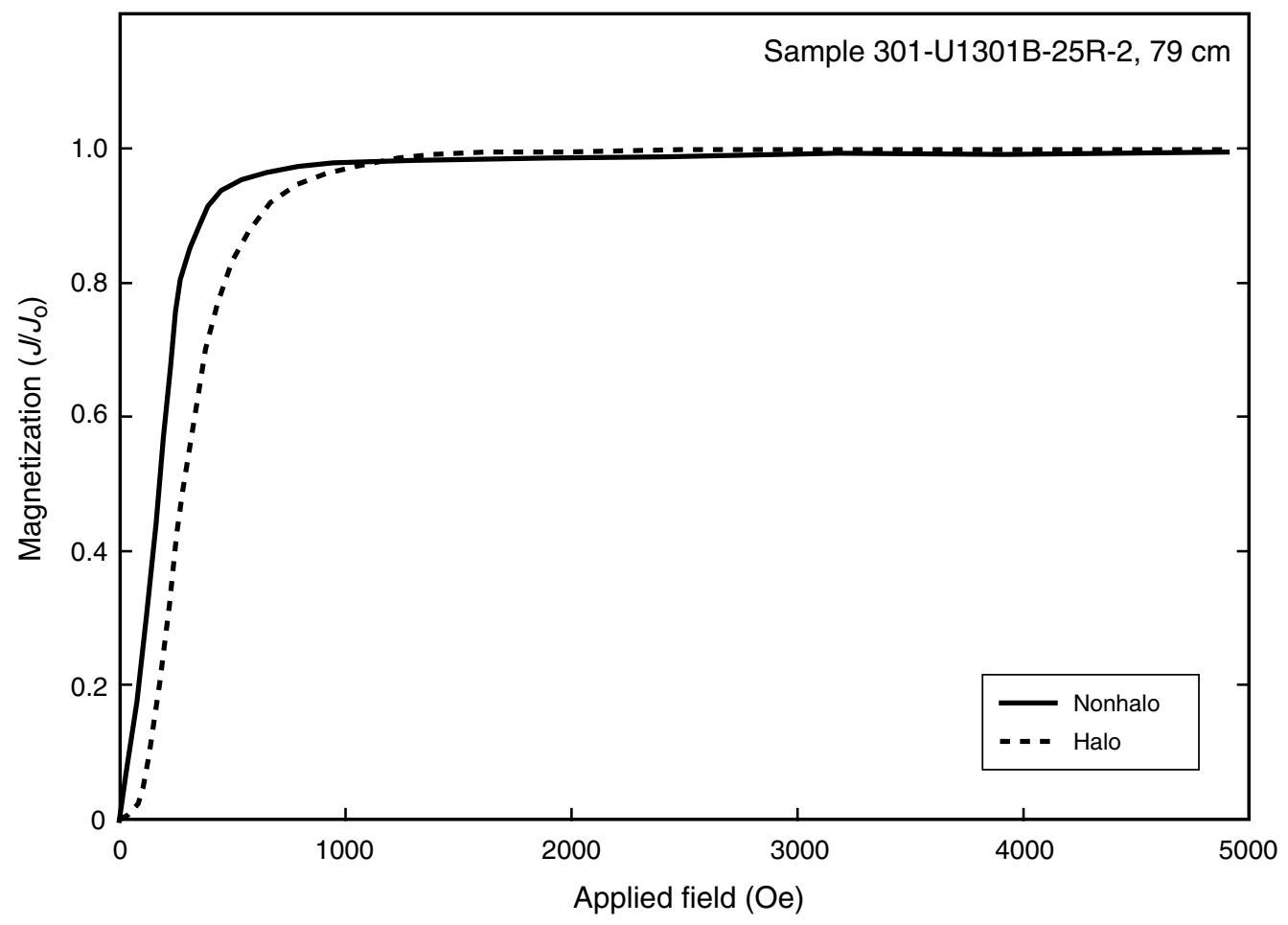


Figure F5. Magnetization behavior upon heating and cooling of two representative basalt samples, Hole U1301B. Sample 301-U1301B-13R-1, $130 \mathrm{~cm}$, shows a reversible curve and gives a Curie temperature (Tc) of $179^{\circ} \mathrm{C}$. Sample 301-U1301B-20R-1, $28 \mathrm{~cm}$, is not reversible upon cooling and gives a Curie temperature of $339^{\circ} \mathrm{C}$.
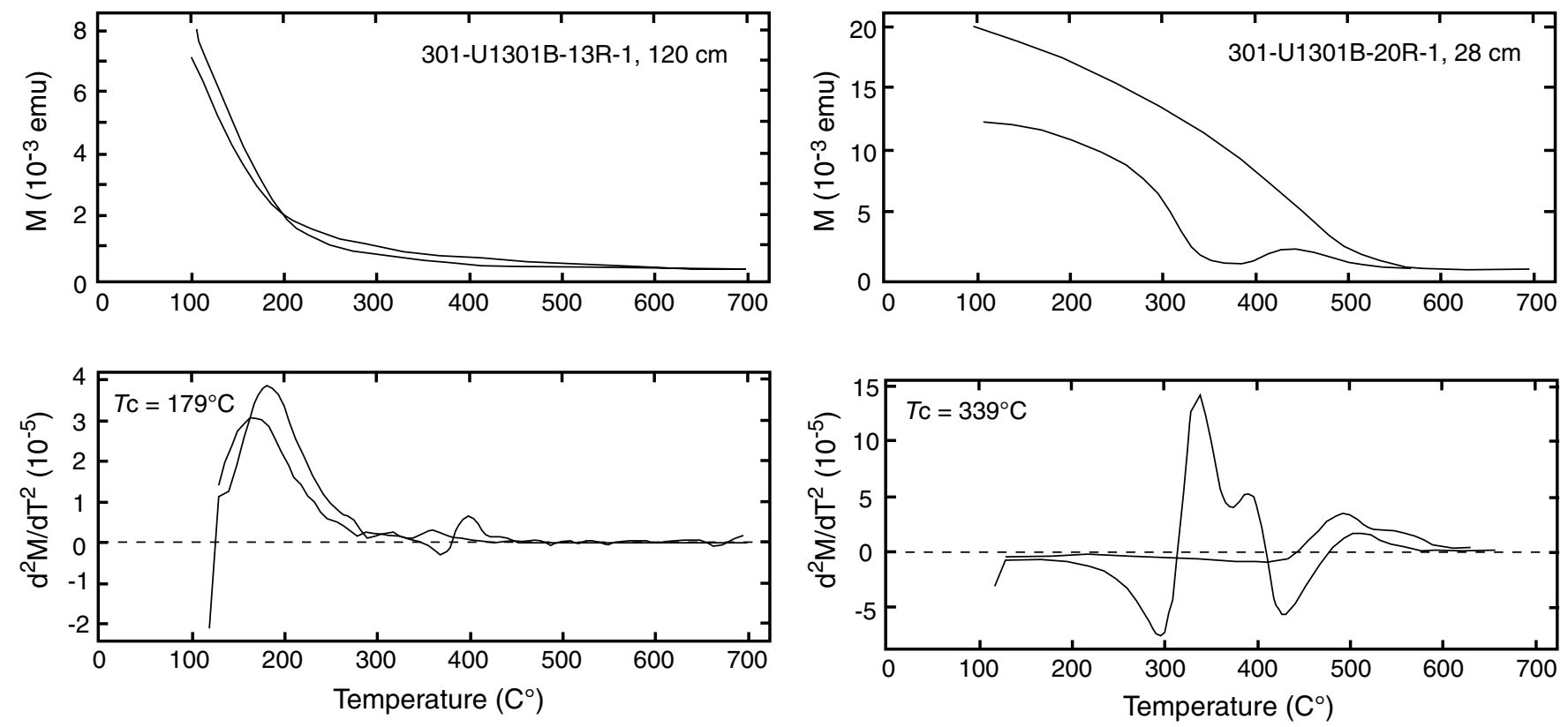
Figure F6. $M_{\mathrm{r}} / M_{\mathrm{s}}$ vs. $H_{\mathrm{cr}} / H_{\mathrm{c}}$ Day plot for basalt samples, Hole U1301B. Vertical and horizontal lines define boundaries of different magnetic grain size fields: $\mathrm{SD}=$ single domain, $\mathrm{PSD}=$ pseudosingle domain, $\mathrm{MD}=$ multidomain. Dotted lines show two example mixing lines for combinations of single and multidomain grains (Dunlop, 2002). Plot axes are logarithmic.

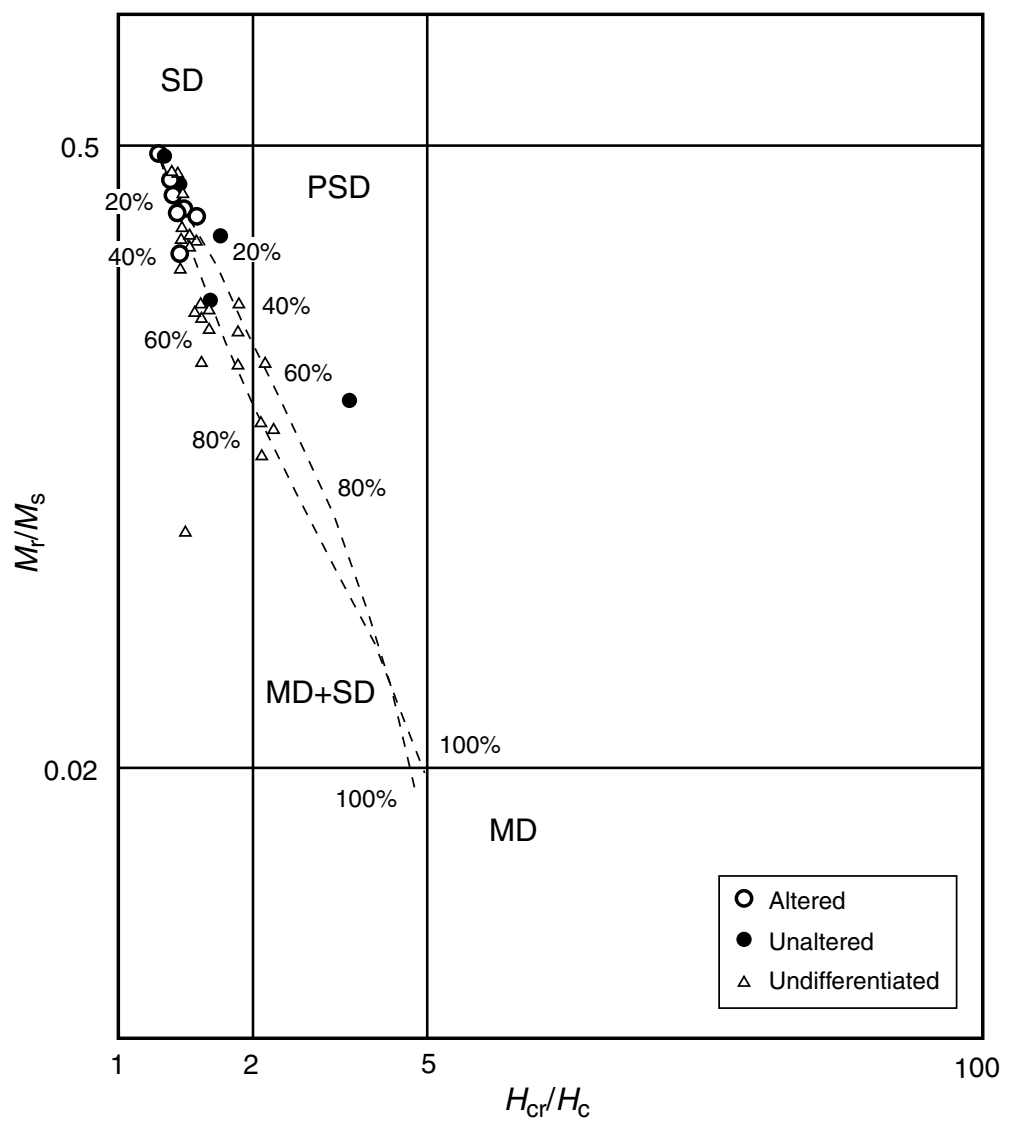


Figure F7. $M_{\mathrm{r}} / M_{\mathrm{s}}$ vs. $H_{\mathrm{cr}} / H_{\mathrm{c}}$ Day plot for basalt samples showing different types of demagnetization behaviors, Hole U1301B (see "NRM and directional measurements" for explanation). SD = single domain, PSD = pseudosingle domain, $\mathrm{MD}=$ multidomain.

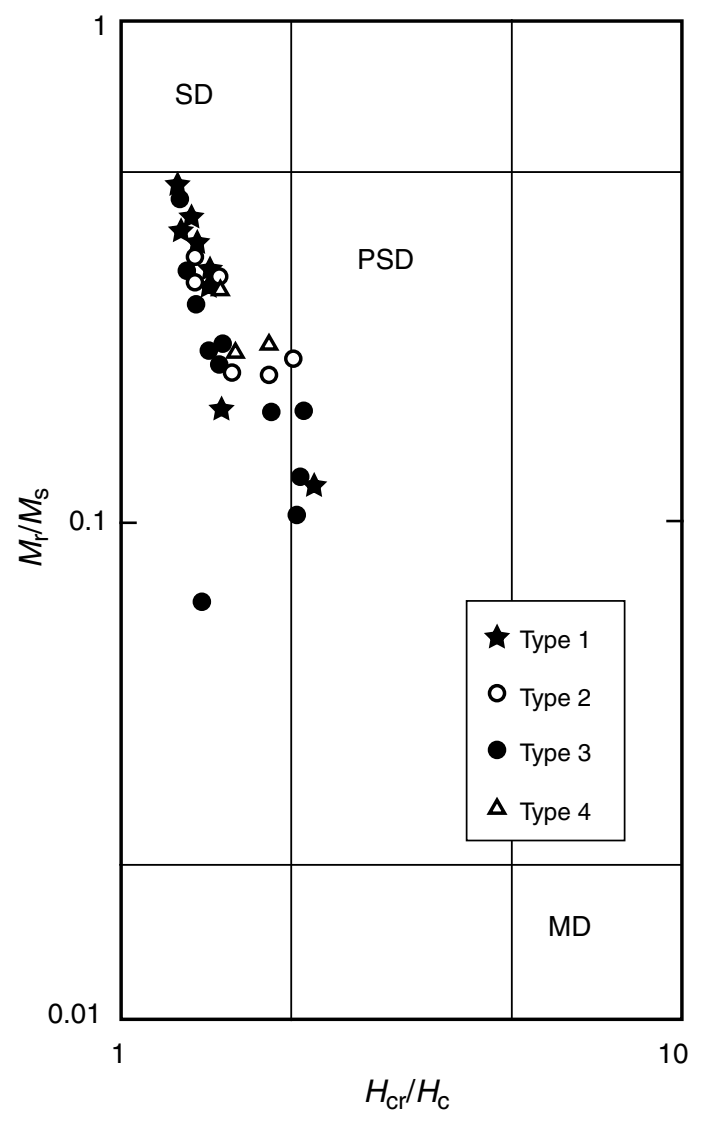


Figure F8. Polished thin section photomicrographs from representative basalt samples, Hole U1301B. Scale bar $=50 \mu \mathrm{m}$. A. Sample 301-U1301B-18R-1, $36 \mathrm{~cm}$, alteration halo. B. Same as A from slightly altered rock. C. Sample 301-U1301B-15R-1, $44 \mathrm{~cm}$, slightly altered.
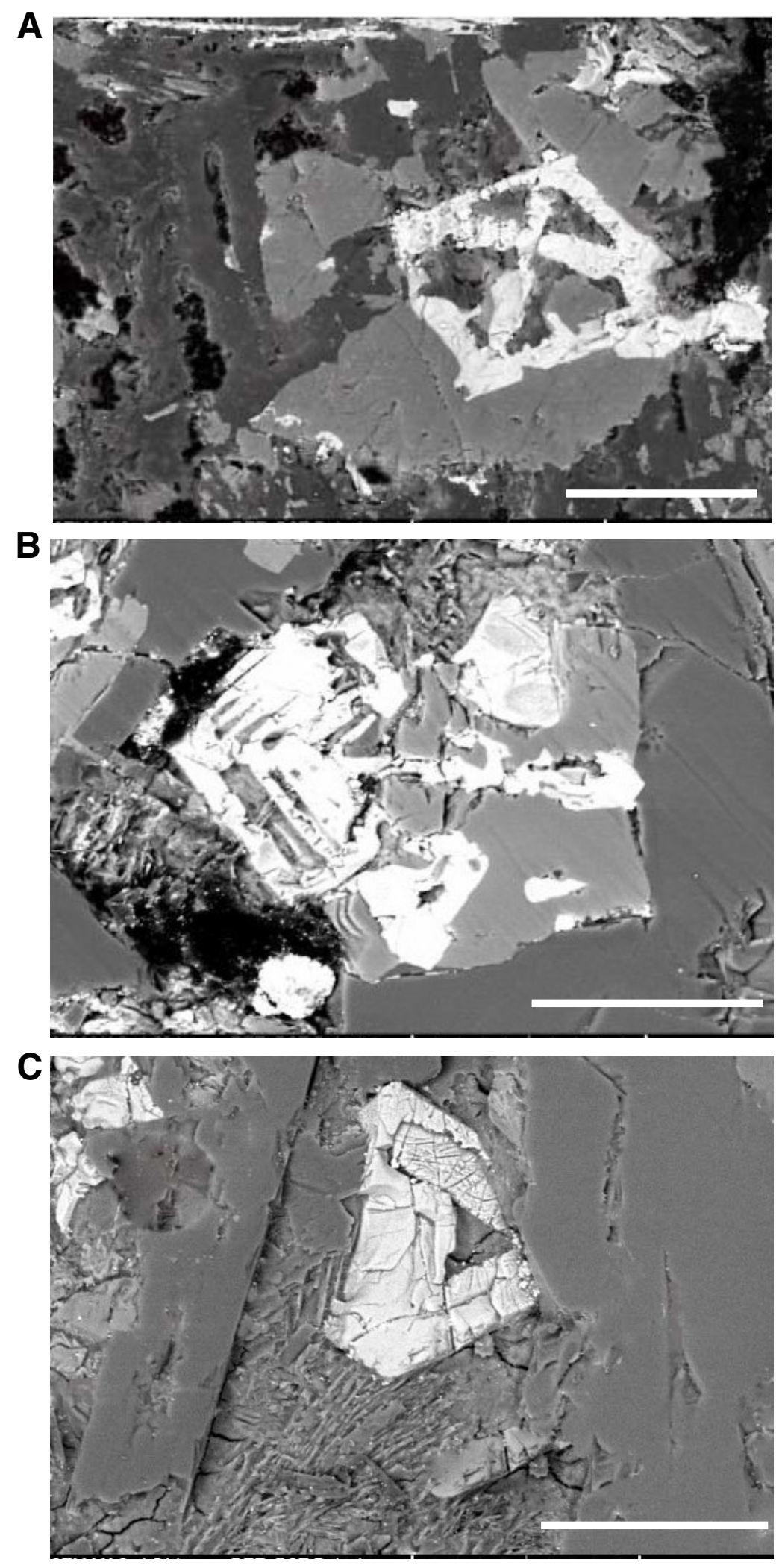
Table T1. Basalt sample paleomagnetic data, Hole U1301B. (See table notes.) (Continued on next four pages.)

\begin{tabular}{|c|c|c|c|c|c|c|c|c|c|c|c|c|}
\hline \multirow{2}{*}{$\begin{array}{l}\text { Core, section, } \\
\text { interval }(\mathrm{cm})\end{array}$} & \multirow{2}{*}{$\begin{array}{l}\text { Depth } \\
\text { (mbsf) }\end{array}$} & \multirow[b]{2}{*}{ Unit } & \multirow{2}{*}{$\begin{array}{l}\text { Unit } \\
\text { type }\end{array}$} & \multirow{2}{*}{$\begin{array}{l}\text { NRM } \\
(A / m)\end{array}$} & \multirow{2}{*}{$\begin{array}{r}\text { Inc. } \\
\left({ }^{\circ}\right)\end{array}$} & \multirow[b]{2}{*}{$N$} & \multirow[b]{2}{*}{ MAD } & & Der & magnetizatio & & \\
\hline & & & & & & & & type & Steps & Treatment & Quality & type \\
\hline 301-U1301B- & & & & & & & & & & & & \\
\hline $1 \mathrm{R}-1,70$ & 351.88 & 1B & $P$ & 5.71 & 63.2 & 5 & 2.8 & AR & $15-35$ & $\mathrm{AF}$ & A & 3 \\
\hline $1 \mathrm{R}-1,72$ & 351.90 & $1 \mathrm{~B}$ & P & 4.51 & 56.0 & 5 & 2.2 & & $300-475$ & $\mathrm{TH}$ & A & 3 \\
\hline $1 \mathrm{R}-1,87$ & 352.07 & 1B & $\mathrm{P}$ & 4.16 & 50.1 & 4 & 2.1 & AR & $25-40$ & AF & A & 3 \\
\hline $1 \mathrm{R}-1,92$ & 352.10 & 1B & $\mathrm{P}$ & 8.22 & 58.2 & 5 & 1.6 & & $300-475$ & $\mathrm{TH}$ & A & 3 \\
\hline $1 \mathrm{R}-1,120$ & 352.40 & 1B & $\mathrm{P}$ & 4.71 & 72.7 & 4 & 0.9 & $A R$ & $15-25$ & $\mathrm{AF}$ & C & 3 \\
\hline $1 \mathrm{R}-1,130$ & 352.50 & 1B & $\mathrm{P}$ & 12.59 & 68.7 & 7 & 1.6 & & $20-50$ & $\mathrm{AF}$ & A & 3 \\
\hline $2 \mathrm{R}-1,17$ & 357.27 & $1 \mathrm{C}$ & $\mathrm{P}$ & 4.72 & 55.1 & 6 & 4.0 & AR & $25-50$ & AF & A & 3 \\
\hline $2 \mathrm{R}-1,30$ & 357.40 & $1 \mathrm{C}$ & $\mathrm{P}$ & 3.16 & 68.3 & 5 & 1.9 & AR & $30-50$ & $\mathrm{AF}$ & A & 3 \\
\hline $2 \mathrm{R}-1,73$ & 357.83 & $1 \mathrm{C}$ & $P$ & 6.90 & 39.3 & 5 & 1.8 & PP & $20-40$ & $\mathrm{AF}$ & C & 2 \\
\hline $2 \mathrm{R}-1,79$ & 357.89 & $1 \mathrm{C}$ & $P$ & 6.81 & 49.2 & 5 & 4.6 & & $300-475$ & $\mathrm{TH}$ & A & 2 \\
\hline $2 \mathrm{R}-1,100$ & 358.10 & $1 \mathrm{C}$ & $\mathrm{P}$ & 3.18 & 52.2 & 4 & 2.1 & AR & $35-50$ & $\mathrm{AF}$ & A & 3 \\
\hline $2 \mathrm{R}-1,100$ & 358.10 & $1 \mathrm{C}$ & $\mathrm{P}$ & 5.07 & 36.8 & 6 & 1.5 & & $400-540$ & $\mathrm{TH}$ & A & 2 \\
\hline $2 \mathrm{R}-1,137$ & 358.47 & $1 \mathrm{C}$ & $\mathrm{P}$ & 11.06 & 39.3 & 8 & 0.9 & & $20-60$ & $\mathrm{AF}$ & C & 2 \\
\hline $2 \mathrm{R}-2,22$ & 358.76 & $1 \mathrm{C}$ & $\mathrm{P}$ & 7.68 & 36.4 & 7 & 2.1 & & $20-50$ & $\mathrm{AF}$ & A & 2 \\
\hline $2 \mathrm{R}-2,110$ & 359.64 & $1 \mathrm{C}$ & $\mathrm{P}$ & 3.00 & 40.2 & 5 & 1.3 & $A R$ & $30-50$ & $\mathrm{AF}$ & A & 2 \\
\hline $2 \mathrm{R}-2,115$ & 359.69 & $1 \mathrm{C}$ & $\mathrm{P}$ & 9.75 & 29.0 & 6 & 1.2 & PP & $15-40$ & $\mathrm{AF}$ & A & 2 \\
\hline $2 \mathrm{R}-3,3$ & 360.07 & $1 \mathrm{C}$ & $\mathrm{P}$ & 8.05 & 39.6 & 4 & 3.4 & & $25-40$ & $\mathrm{AF}$ & C & 2 \\
\hline $2 \mathrm{R}-3,95$ & 360.99 & $1 \mathrm{C}$ & $\mathrm{P}$ & 7.02 & 50.1 & 7 & 9.3 & & $350-550$ & $\mathrm{TH}$ & A & 3 \\
\hline $2 \mathrm{R}-3,123$ & 361.27 & $1 \mathrm{C}$ & $P$ & 12.57 & 45.6 & 6 & 1.0 & PP & $20-50$ & $\mathrm{AF}$ & A & 2 \\
\hline $3 R-3,130$ & 361.34 & $1 \mathrm{C}$ & $\mathrm{P}$ & 2.95 & 41.9 & 5 & 1.7 & $A R$ & $30-50$ & $\mathrm{AF}$ & A & 2 \\
\hline $3 \mathrm{R}-1,24$ & 361.34 & $1 \mathrm{C}$ & $P$ & 8.09 & 48.0 & 3 & 4.9 & & $300-450$ & $\mathrm{TH}$ & A & 3 \\
\hline $3 R-1,30$ & 361.40 & $1 \mathrm{C}$ & $P$ & 4.41 & 58.7 & 5 & 1.4 & $A R$ & $20-40$ & AF & A & 3 \\
\hline $3 \mathrm{R}-1,31$ & 361.41 & $1 \mathrm{C}$ & $\mathrm{P}$ & 6.90 & 42.4 & 6 & 0.4 & PP & $15-40$ & $\mathrm{AF}$ & A & 2 \\
\hline $3 \mathrm{R}-1,112$ & 362.22 & $1 \mathrm{C}$ & $\mathrm{P}$ & 3.88 & 51.2 & 6 & 0.7 & AR & $25-50$ & $\mathrm{AF}$ & A & 3 \\
\hline $3 \mathrm{R}-1,130$ & 362.40 & $1 \mathrm{C}$ & $P$ & 8.70 & 49.2 & 5 & 0.7 & & $15-35$ & $\mathrm{AF}$ & B & 3 \\
\hline $3 R-1,135$ & 362.45 & $1 \mathrm{C}$ & $\mathrm{P}$ & 4.80 & 45.7 & 6 & 0.9 & AR & $25-50$ & $\mathrm{AF}$ & A & 2 \\
\hline $3 \mathrm{R}-2,10$ & 362.68 & $1 \mathrm{C}$ & $\mathrm{P}$ & 3.79 & 61.9 & 5 & 1.9 & AR & $30-50$ & AF & A & 3 \\
\hline $3 R-2,12$ & 362.70 & $1 \mathrm{C}$ & $\mathrm{P}$ & 11.49 & $-54.6^{*}$ & 5 & 0.5 & & $250-450$ & TH & A & 3 \\
\hline $3 R-2,58$ & 363.18 & $1 \mathrm{C}$ & $P$ & 10.00 & 25.8 & 6 & 0.5 & PP & $20-50$ & $\mathrm{AF}$ & A & 2 \\
\hline $3 R-2,88$ & 363.48 & $1 \mathrm{C}$ & $\mathrm{P}$ & 10.37 & 44.8 & 5 & 0.4 & & $20-40$ & $\mathrm{AF}$ & A & 2 \\
\hline $3 R-2,107$ & 363.67 & $1 \mathrm{C}$ & $\mathrm{P}$ & 5.60 & 52.8 & 5 & 0.5 & $A R$ & $30-50$ & $\mathrm{AF}$ & A & 3 \\
\hline $4 \mathrm{R}-1,37$ & 366.97 & $1 \mathrm{C}$ & $\mathrm{P}$ & 11.74 & 54.3 & 4 & 0.1 & & $250-450$ & $\mathrm{TH}$ & A & 3 \\
\hline $4 \mathrm{R}-1,51$ & 366.11 & $1 \mathrm{C}$ & $\mathrm{P}$ & 9.96 & 39.5 & 5 & 1.4 & & $420-540$ & $\mathrm{TH}$ & A & 2 \\
\hline $4 \mathrm{R}-1,55$ & 367.15 & $1 \mathrm{C}$ & $\mathrm{P}$ & 6.88 & 71.3 & 4 & 0.8 & AR & $35-50$ & $\mathrm{AF}$ & C & 3 \\
\hline $4 \mathrm{R}-1,110$ & 367.70 & $1 \mathrm{C}$ & $\mathrm{P}$ & 9.98 & 40.5 & 5 & 1.3 & PP & $25-45$ & $\mathrm{AF}$ & A & 2 \\
\hline $4 \mathrm{R}-1,134$ & 367.94 & $1 \mathrm{C}$ & $\mathrm{P}$ & 8.59 & 41.0 & 4 & 10.0 & & $350-500$ & $\mathrm{TH}$ & A & 2 \\
\hline $4 \mathrm{R}-2,20$ & 368.26 & $1 \mathrm{C}$ & $P$ & 6.06 & 59.7 & 4 & 2.0 & AR & $40-60$ & $\mathrm{AF}$ & C & 3 \\
\hline $4 \mathrm{R}-2,23$ & 368.29 & $1 \mathrm{C}$ & $\mathrm{P}$ & 10.70 & 66.4 & 4 & 1.1 & & $20-35$ & $\mathrm{AF}$ & A & 3 \\
\hline $4 \mathrm{R}-2,84$ & 368.90 & $1 \mathrm{C}$ & $\mathrm{P}$ & 4.45 & 42.4 & 5 & 0.6 & AR & $30-50$ & $\mathrm{AF}$ & A & 2 \\
\hline $4 \mathrm{R}-2,88$ & 368.94 & $1 \mathrm{C}$ & P & 9.41 & 35.8 & 5 & 1.8 & & $250-500$ & $\mathrm{TH}$ & A & 2 \\
\hline 4R-3, 1 & 369.40 & $1 \mathrm{C}$ & $\mathrm{P}$ & 3.58 & 43.9 & 5 & 1.5 & $A R$ & $35-60$ & $\mathrm{AF}$ & A & 2 \\
\hline $4 \mathrm{R}-3,25$ & 369.24 & $1 \mathrm{C}$ & $\mathrm{P}$ & 12.18 & 47.2 & 4 & 2.2 & & $300-500$ & $\mathrm{TH}$ & A & 2 \\
\hline $4 \mathrm{R}-3,27$ & 369.66 & $1 \mathrm{C}$ & P & 9.07 & 51.3 & 6 & 2.8 & & $25-60$ & AF & A & 3 \\
\hline $4 R-3,64$ & 370.03 & $1 \mathrm{C}$ & $P$ & 7.33 & 63.1 & 5 & 2.2 & $A R$ & $30-50$ & $\mathrm{AF}$ & B & 3 \\
\hline $4 \mathrm{R}-3,78$ & 370.17 & $1 \mathrm{C}$ & $\mathrm{P}$ & 14.55 & 40.9 & 5 & 1.1 & & $25-45$ & $\mathrm{AF}$ & B & 2 \\
\hline $4 \mathrm{R}-3,132$ & 370.71 & $1 \mathrm{C}$ & $\mathrm{P}$ & 14.61 & 49.9 & 5 & 1.7 & PP & $20-40$ & AF & A & 2 \\
\hline $4 \mathrm{R}-4,45$ & 371.29 & $1 \mathrm{C}$ & $\mathrm{P}$ & 13.94 & 63.5 & 4 & 1.1 & & $200-400$ & $\mathrm{TH}$ & B & 3 \\
\hline $5 R-1,37$ & 376.67 & $1 \mathrm{C}$ & $\mathrm{P}$ & 8.48 & 53.2 & 6 & 1.4 & & $25-60$ & $\mathrm{AF}$ & A & 3 \\
\hline $5 R-1,57$ & 376.87 & $1 \mathrm{C}$ & P & 4.47 & 24.0 & 4 & 0.5 & AR & $40-60$ & $\mathrm{AF}$ & A & 2 \\
\hline $5 \mathrm{R}-1,100$ & 377.30 & $1 \mathrm{C}$ & P & 2.08 & 56.0 & 5 & 2.7 & $A R$ & $35-60$ & $\mathrm{AF}$ & A & 3 \\
\hline $5 \mathrm{R}-1,100$ & 377.30 & $1 \mathrm{C}$ & P & 5.45 & 36.4 & 6 & 0.6 & & $350-520$ & $\mathrm{TH}$ & A & 2 \\
\hline $5 \mathrm{R}-2,27$ & 378.07 & $1 \mathrm{C}$ & $P$ & 1.69 & 69.4 & 5 & 0.5 & AR & $30-50$ & AF & A & 3 \\
\hline $5 R-2,104$ & 378.84 & $1 \mathrm{C}$ & $\mathrm{P}$ & 16.65 & 35.8 & 6 & 3.5 & PP & $30-50$ & AF & A & 2 \\
\hline $5 R-2,105$ & 378.85 & $1 \mathrm{C}$ & $\mathrm{P}$ & 1.92 & 60.8 & 5 & 2.0 & AR & $35-60$ & $\mathrm{AF}$ & A & 3 \\
\hline $5 \mathrm{R}-2,109$ & 378.89 & $1 \mathrm{C}$ & $\mathrm{P}$ & 16.49 & 53.6 & 7 & 1.9 & & $275-475$ & $\mathrm{TH}$ & B & 3 \\
\hline $5 R-3,44$ & 379.74 & $1 \mathrm{C}$ & $\mathrm{P}$ & 28.52 & 59.7 & 4 & 12.6 & & $375-475$ & $\mathrm{TH}$ & A & 3 \\
\hline $5 R-3,55$ & 379.85 & $1 \mathrm{C}$ & $\mathrm{P}$ & 2.69 & 45.9 & 5 & 1.6 & AR & $35-60$ & AF & A & 2 \\
\hline $6 \mathrm{R}-1,33$ & 386.33 & $1 \mathrm{C}$ & $P$ & 12.49 & 67.8 & 5 & 2.7 & & $150-350$ & $\mathrm{TH}$ & B & 3 \\
\hline $6 \mathrm{R}-1,41$ & 386.41 & $1 C$ & $\mathrm{P}$ & 15.96 & 81.9 & 4 & 1.5 & PP & $25-40$ & $\mathrm{AF}$ & C & 3 \\
\hline $6 \mathrm{R}-1,48$ & 386.48 & $1 \mathrm{C}$ & $\mathrm{P}$ & 5.78 & 67.4 & 3 & 1.6 & AR & $45-60$ & $\mathrm{AF}$ & B & 3 \\
\hline $6 \mathrm{R}-1,64$ & 386.64 & $1 \mathrm{C}$ & $\mathrm{P}$ & 12.53 & 59.6 & 4 & 2.4 & & $35-60$ & $\mathrm{AF}$ & B & 3 \\
\hline $6 \mathrm{R}-1,86$ & 386.86 & $1 \mathrm{C}$ & $\mathrm{P}$ & 4.59 & 76.8 & 4 & 1.5 & $A R$ & $30-45$ & $\mathrm{AF}$ & B & 4 \\
\hline $6 \mathrm{R}-1,92$ & 386.92 & $1 \mathrm{C}$ & $\mathrm{P}$ & 4.81 & 83.2 & 6 & 1.7 & $A R$ & $30-60$ & $\mathrm{AF}$ & B & 4 \\
\hline $6 \mathrm{R}-2,4$ & 387.49 & $1 \mathrm{C}$ & P & 4.28 & 66.0 & 5 & 3.3 & AR & $35-60$ & $\mathrm{AF}$ & A & 3 \\
\hline $6 \mathrm{R}-2,35$ & 387.80 & $1 \mathrm{C}$ & $\mathrm{P}$ & 17.24 & $-56.7^{\star}$ & 5 & 0.4 & PP & $35-60$ & $\mathrm{AF}$ & A & 3 \\
\hline $6 \mathrm{R}-2,44$ & 387.89 & $1 \mathrm{C}$ & $P$ & 4.74 & 78.2 & 5 & 2.5 & AR & $35-60$ & $\mathrm{AF}$ & A & 3 \\
\hline $6 \mathrm{R}-2,48$ & 387.93 & $1 \mathrm{C}$ & $\mathrm{P}$ & 5.07 & 59.5 & 5 & 4.6 & & $420-540$ & $\mathrm{TH}$ & A & 3 \\
\hline
\end{tabular}


Table T1 (continued). (Continued on next page.)

\begin{tabular}{|c|c|c|c|c|c|c|c|c|c|c|c|c|}
\hline \multirow{2}{*}{$\begin{array}{l}\text { Core, section, } \\
\text { interval }(\mathrm{cm})\end{array}$} & \multirow{2}{*}{$\begin{array}{l}\text { Depth } \\
\text { (mbsf) }\end{array}$} & \multirow[b]{2}{*}{ Unit } & \multirow{2}{*}{$\begin{array}{l}\text { Unit } \\
\text { type }\end{array}$} & \multirow{2}{*}{$\begin{array}{l}\text { NRM } \\
(\mathrm{A} / \mathrm{m})\end{array}$} & \multirow{2}{*}{$\begin{array}{c}\text { Inc. } \\
\left({ }^{\circ}\right)\end{array}$} & \multirow[b]{2}{*}{$N$} & \multirow[b]{2}{*}{ MAD } & Sample & Den & nagnetizatic & & ChRM \\
\hline & & & & & & & & type & Steps & Treatment & Quality & type \\
\hline $6 \mathrm{R}-2,67$ & 388.12 & $1 \mathrm{C}$ & $\mathrm{P}$ & 10.22 & 66.8 & 4 & 1.2 & & $350-475$ & $\mathrm{TH}$ & A & 3 \\
\hline 7R-1, 29 & 395.89 & $1 \mathrm{C}$ & $P$ & 14.67 & 62.2 & 6 & 0.9 & & $25-60$ & $\mathrm{AF}$ & C & 3 \\
\hline $7 R-1,30$ & 395.90 & $1 C$ & $P$ & 60.81 & 63.8 & 6 & 1.6 & $A R$ & $30-60$ & $\mathrm{AF}$ & A & 3 \\
\hline $7 \mathrm{R}-1,52$ & 396.12 & $1 \mathrm{C}$ & $P$ & 9.26 & -6.9 & 4 & 1.7 & & $150-275$ & $\mathrm{TH}$ & A & 1 \\
\hline $7 R-1,57$ & 396.17 & $1 \mathrm{C}$ & $P$ & 6.68 & -45.6 & 4 & 3.5 & AR & $35-50$ & $\mathrm{AF}$ & A & 1 \\
\hline $7 R-2,4$ & 397.14 & $1 \mathrm{C}$ & $\mathrm{P}$ & 10.90 & 42.6 & 4 & 12.8 & PP & $40-60$ & $\mathrm{AF}$ & C & 2 \\
\hline $8 \mathrm{R}-1,19$ & 405.39 & $1 \mathrm{C}$ & $\mathrm{P}$ & 6.90 & -59.7 & 5 & 1.8 & & $35-60$ & $\mathrm{AF}$ & B & 1 \\
\hline $8 \mathrm{R}-1,43$ & 405.63 & $1 C$ & $\mathrm{P}$ & 6.26 & 37.7 & 6 & 0.3 & AR & $30-60$ & $\mathrm{AF}$ & A & 2 \\
\hline $8 \mathrm{R}-1,44$ & 405.64 & $1 C$ & $P$ & 8.79 & 47.7 & 6 & 1.0 & & $30-60$ & $\mathrm{AF}$ & A & 2 \\
\hline $8 R-1,63$ & 405.83 & $1 C$ & $P$ & 5.78 & -2.8 & 3 & 1.9 & $A R$ & $45-60$ & $\mathrm{AF}$ & C & 1 \\
\hline $9 \mathrm{R}-1,30$ & 410.50 & $1 C$ & $P$ & 10.76 & 57.3 & 4 & 10.3 & & $425-500$ & $\mathrm{TH}$ & A & 3 \\
\hline $9 \mathrm{R}-1,32$ & 410.52 & $1 C$ & $P$ & 4.69 & 36.1 & 4 & 2.9 & AR & $40-60$ & $\mathrm{AF}$ & $A$ & 2 \\
\hline $9 R-1,53$ & 410.73 & $1 C$ & $P$ & 8.03 & 57.6 & 5 & 0.4 & AR & $35-60$ & $\mathrm{AF}$ & A & 3 \\
\hline $9 R-1,63$ & 410.03 & $1 C$ & $\mathrm{P}$ & 13.78 & 75.5 & 5 & 7.9 & & $400-500$ & $\mathrm{TH}$ & $A$ & 4 \\
\hline $9 R-1,63$ & 410.03 & $1 C$ & $\mathrm{P}$ & 4.79 & 62.4 & 4 & 0.8 & AR & $30-45$ & $\mathrm{AF}$ & A & 3 \\
\hline $10 \mathrm{R}-1,26$ & 415.06 & $1 C$ & $\mathrm{P}$ & 6.18 & 49.8 & 5 & 0.7 & AR & $35-60$ & $\mathrm{AF}$ & A & 2 \\
\hline $10 \mathrm{R}-1,45$ & 415.25 & $1 \mathrm{C}$ & $\mathrm{P}$ & 7.37 & 46.5 & 3 & 2.0 & & $30-40$ & $\mathrm{AF}$ & C & 2 \\
\hline 10R-1, 79 & 415.59 & $1 \mathrm{C}$ & $\mathrm{P}$ & 9.11 & 61.8 & 5 & 8.7 & & $400-500$ & $\mathrm{TH}$ & A & 3 \\
\hline $10 R-1,95$ & 415.75 & $1 \mathrm{C}$ & $\mathrm{P}$ & 6.05 & 73.6 & 5 & 5.7 & & $400-500$ & $\mathrm{TH}$ & A & 3 \\
\hline 10R-1, 131 & 416.11 & $1 \mathrm{C}$ & $\mathrm{P}$ & 13.04 & 64.5 & 6 & 4.5 & & $375-500$ & $\mathrm{TH}$ & A & 3 \\
\hline $10 \mathrm{R}-2,57$ & 416.82 & $1 \mathrm{C}$ & $\mathrm{P}$ & 14.02 & 64.8 & 4 & 1.5 & & $35-50$ & $\mathrm{AF}$ & A & 3 \\
\hline $10 \mathrm{R}-2,67$ & 415.92 & $1 C$ & $\mathrm{P}$ & 4.73 & 45.5 & 5 & 1.1 & AR & $35-60$ & $\mathrm{AF}$ & C & 2 \\
\hline $10 \mathrm{R}-2,96$ & 417.21 & $1 C$ & $P$ & 6.62 & 66.7 & 4 & 2.4 & AR & $40-60$ & $\mathrm{AF}$ & C & 4 \\
\hline $11 \mathrm{R}-1,67$ & 425.07 & $2 \mathrm{~A}$ & $M$ & 5.14 & 76.7 & 4 & 1.2 & AR & $40-60$ & $\mathrm{AF}$ & A & 4 \\
\hline $11 R-1,68$ & 425.08 & $2 \mathrm{~A}$ & M & 13.96 & 84.5 & 4 & 3.4 & & $425-500$ & $\mathrm{TH}$ & B & 4 \\
\hline $11 \mathrm{R}-1,94$ & 425.34 & $2 \mathrm{~A}$ & M & 34.41 & 35.0 & 3 & 6.3 & & $450-500$ & $\mathrm{TH}$ & $A$ & 2 \\
\hline 11R-1, 107 & 425.47 & $2 \mathrm{~A}$ & $M$ & 5.36 & 49.3 & 5 & 1.5 & $\mathrm{AR}$ & $30-45$ & $\mathrm{AF}$ & B & 2 \\
\hline $12 \mathrm{R}-1,27$ & 429.17 & $2 \mathrm{~A}$ & $M$ & 25.58 & 32.7 & 6 & 8.8 & & $300-500$ & $\mathrm{TH}$ & A & 2 \\
\hline $12 \mathrm{R}-1,70$ & 429.60 & $2 \mathrm{~A}$ & $M$ & 19.28 & 83.5 & 5 & 2.2 & & $190-235$ & $\mathrm{TH}$ & C & 5 \\
\hline 12R-1, 91 & 429.81 & $2 \mathrm{~A}$ & $\mathrm{M}$ & 28.52 & 69.7 & 6 & 3.8 & & $30-60$ & $\mathrm{AF}$ & A & 3 \\
\hline 12R-1, 93 & 429.83 & $2 \mathrm{~A}$ & $\mathrm{M}$ & 30.58 & 64.1 & 6 & 2.9 & & $30-60$ & $\mathrm{AF}$ & A & 3 \\
\hline $12 \mathrm{R}-1,100$ & 429.90 & $2 \mathrm{~A}$ & $\mathrm{M}$ & 51.14 & 59.9 & 5 & 2.6 & & $25-45$ & $\mathrm{AF}$ & A & 3 \\
\hline 13R-1, 14 & 430.04 & $2 \mathrm{~A}$ & $\mathrm{M}$ & 6.06 & 57.1 & 5 & 2.7 & AR & $35-60$ & $\mathrm{AF}$ & A & 3 \\
\hline $13 \mathrm{R}-1,22$ & 430.12 & $2 \mathrm{~A}$ & M & 28.60 & 63.1 & 4 & 12.4 & & $400-475$ & $\mathrm{TH}$ & B & 3 \\
\hline $13 \mathrm{R}-1,45$ & 430.35 & $2 \mathrm{~A}$ & $\mathrm{M}$ & 60.35 & 75.4 & 4 & 3.3 & & $250-300$ & $\mathrm{TH}$ & A & 3 \\
\hline $13 R-1,68$ & 430.58 & $2 \mathrm{~A}$ & $\mathrm{M}$ & 32.17 & 70.1 & 5 & 6.3 & & $30-50$ & $\mathrm{AF}$ & A & 3 \\
\hline 13R-1, 94 & 430.84 & $2 \mathrm{~A}$ & $\mathrm{M}$ & 2.80 & 70.3 & 5 & 1.1 & AR & $30-50$ & $\mathrm{AF}$ & A & 3 \\
\hline $13 \mathrm{R}-1,120$ & 431.10 & $2 \mathrm{~A}$ & $M$ & 70.27 & 85.3 & 5 & 5.1 & & $300-420$ & $\mathrm{TH}$ & $A$ & 5 \\
\hline $13 R-2,5$ & 431.30 & $2 \mathrm{~A}$ & $\mathrm{M}$ & 17.70 & 63.8 & 4 & 13.8 & & $350-450$ & $\mathrm{TH}$ & A & 3 \\
\hline $13 \mathrm{R}-2,14$ & 431.47 & $2 \mathrm{~A}$ & M & 4.18 & 84.2 & 3 & 0.6 & AR & $40-50$ & $\mathrm{AF}$ & C & 4 \\
\hline $13 \mathrm{R}-2,17$ & 431.50 & $2 \mathrm{~A}$ & M & 1.33 & & & & $\mathrm{AR}$ & & $\mathrm{AF}$ & & \\
\hline $14 \mathrm{R}-1,26$ & 434.26 & $2 B$ & $\mathrm{M}$ & 6.88 & 66.5 & 4 & 0.4 & AR & $30-45$ & $\mathrm{AF}$ & B & 3 \\
\hline $14 \mathrm{R}-1,45$ & 434.45 & $2 \mathrm{~B}$ & $\mathrm{M}$ & 4.75 & 49.2 & 4 & 8.7 & AR & $35-50$ & $\mathrm{AF}$ & C & 2 \\
\hline $14 \mathrm{R}-1,55$ & 434.55 & $2 B$ & $\mathrm{M}$ & 6.08 & 48.8 & 5 & 7.2 & AR & $30-50$ & $\mathrm{AF}$ & A & 2 \\
\hline 14R-1, 81 & 434.81 & $2 B$ & $\mathrm{M}$ & 25.51 & 54.0 & 5 & 4.8 & & $30-50$ & $\mathrm{AF}$ & A & 4 \\
\hline 14R-1, 132 & 435.32 & 3 & $P$ & 13.73 & 77.6 & 4 & 1.4 & & $250-375$ & $\mathrm{TH}$ & $\mathrm{D}$ & 4 \\
\hline 14R-1, 133 & 435.33 & 3 & $\mathrm{P}$ & 7.13 & 66.9 & 3 & 1.3 & $A R$ & $45-60$ & $\mathrm{AF}$ & A & 3 \\
\hline 14R-1, 142 & 435.42 & 3 & $P$ & 3.61 & 84.3 & 4 & 0.7 & $A R$ & $35-50$ & $\mathrm{AF}$ & B & 4 \\
\hline $15 R-1,45$ & 444.05 & 3 & $\mathrm{P}$ & 6.61 & 54.2 & 4 & 1.1 & AR & $35-50$ & $\mathrm{AF}$ & A & 3 \\
\hline 15R-1, 94 & 444.54 & $4 \mathrm{~A}$ & $\mathrm{M}$ & 11.58 & 61.6 & 3 & 5.0 & & $425-500$ & $\mathrm{TH}$ & A & 3 \\
\hline 15R-1, 108 & 444.68 & $4 B$ & M & 15.33 & 55.1 & 4 & 12.1 & PP & $35-50$ & $\mathrm{AF}$ & B & 3 \\
\hline 15R-1, 113 & 444.73 & $4 \mathrm{~B}$ & $\mathrm{M}$ & 16.83 & 62.5 & 7 & 1.8 & & $20-50$ & $\mathrm{AF}$ & A & 3 \\
\hline $15 R-2,9$ & 445.19 & $4 B$ & $M$ & 27.40 & 63.2 & 6 & 5.2 & & $20-45$ & $\mathrm{AF}$ & A & 3 \\
\hline $15 R-2,20$ & 445.30 & $4 B$ & $\mathrm{M}$ & 23.98 & 62.4 & 4 & 8.3 & PP & $20-35$ & $\mathrm{AF}$ & A & 3 \\
\hline $15 R-2,70$ & 445.80 & $4 B$ & $\mathrm{M}$ & 35.93 & 63.8 & 3 & 5.6 & & $380-460$ & $\mathrm{TH}$ & B & 3 \\
\hline $15 R-2,78$ & 445.88 & $4 B$ & $\mathrm{M}$ & 33.91 & 38.1 & 2 & 3.0 & & $460-515$ & $\mathrm{TH}$ & $\mathrm{B}^{\dagger}$ & 2 \\
\hline 15R-2, 91 & 446.01 & $4 \mathrm{~B}$ & $\mathrm{M}$ & 27.89 & 55.1 & 6 & 14.4 & & $350-475$ & $\mathrm{TH}$ & A & 3 \\
\hline 15R-2, 93 & 446.03 & $4 \mathrm{~B}$ & $\mathrm{M}$ & 30.91 & 43.0 & 5 & 4.7 & & $30-50$ & $\mathrm{AF}$ & A & 2 \\
\hline $15 R-2,127$ & 446.37 & $4 \mathrm{~B}$ & $\mathrm{M}$ & 70.31 & 78.8 & 4 & 1.4 & & $335-420$ & $\mathrm{TH}$ & B & 3 \\
\hline 15R-3, 10 & 446.70 & $4 B$ & M & 24.62 & 30.5 & 5 & 3.7 & & $35-60$ & $\mathrm{AF}$ & A & 2 \\
\hline $15 R-3,22$ & 446.82 & $4 B$ & $\mathrm{M}$ & 5.76 & 48.7 & 3 & 1.3 & AR & $35-45$ & $\mathrm{AF}$ & C & 2 \\
\hline $15 R-3,24$ & 446.84 & $4 B$ & $M$ & 52.63 & 52.1 & 4 & 3.6 & & $460-540$ & $\mathrm{TH}$ & A & 2 \\
\hline 15R-3, 41 & 447.01 & $4 B$ & $\mathrm{M}$ & 5.26 & 51.0 & 4 & 0.6 & $A R$ & $40-60$ & $\mathrm{AF}$ & A & 3 \\
\hline $15 R-3,63$ & 447.23 & $4 B$ & $M$ & 43.61 & 74.9 & 6 & 7.1 & & $95-180$ & $\mathrm{AF}$ & A & 3 \\
\hline 15R-3, 81 & 447.41 & $4 B$ & $M$ & 39.73 & 66.4 & 5 & 1.2 & & $35-60$ & $\mathrm{AF}$ & A & 3 \\
\hline $15 R-3,120$ & 447.80 & $4 B$ & $\mathrm{M}$ & 41.45 & 84.7 & 3 & 1.3 & & $370-450$ & $\mathrm{TH}$ & B & 5 \\
\hline $15 R-3,144$ & 448.04 & $4 B$ & $M$ & 6.58 & 51.2 & 4 & 1.8 & PP & $30-45$ & $\mathrm{AF}$ & B & 3 \\
\hline $15 R-4,5$ & 448.15 & $4 C$ & $M$ & 3.67 & 48.1 & 5 & 0.4 & $A R$ & $35-60$ & $\mathrm{AF}$ & B & 2 \\
\hline
\end{tabular}


Table T1 (continued). (Continued on next page.)

\begin{tabular}{|c|c|c|c|c|c|c|c|c|c|c|c|c|}
\hline \multirow{2}{*}{$\begin{array}{l}\text { Core, section, } \\
\text { interval }(\mathrm{cm})\end{array}$} & \multirow{2}{*}{$\begin{array}{l}\text { Depth } \\
\text { (mbsf) }\end{array}$} & \multirow[b]{2}{*}{ Unit } & \multirow{2}{*}{$\begin{array}{l}\text { Unit } \\
\text { type }\end{array}$} & \multirow{2}{*}{$\begin{array}{l}\text { NRM } \\
(\mathrm{A} / \mathrm{m})\end{array}$} & \multirow{2}{*}{$\begin{array}{c}\text { Inc. } \\
\left({ }^{\circ}\right)\end{array}$} & \multirow[b]{2}{*}{$N$} & \multirow[b]{2}{*}{ MAD } & & Den & nagnetizatic & & ChRM \\
\hline & & & & & & & & type & Steps & Treatment & Quality & type \\
\hline 15R-4, 21 & 448.31 & $4 C$ & $\mathrm{M}$ & 23.39 & 46.3 & 4 & 9.2 & & $25-40$ & $\mathrm{AF}$ & A & 2 \\
\hline $15 R-4,29$ & 448.39 & $4 C$ & $\mathrm{M}$ & 3.30 & $-72.6^{*}$ & 4 & 3.0 & $A R$ & $35-50$ & $\mathrm{AF}$ & A & 4 \\
\hline $15 R-4,30$ & 448.40 & $4 C$ & $M$ & 17.24 & 73.4 & 4 & 2.6 & & $370-500$ & $\mathrm{TH}$ & A & 3 \\
\hline $15 R-4,65$ & 448.75 & $4 C$ & $\mathrm{M}$ & 15.99 & 67.2 & 5 & 3.9 & PP & $20-45$ & $\mathrm{AF}$ & A & 3 \\
\hline 15R-4, 101 & 449.11 & $4 C$ & $\mathrm{M}$ & 36.80 & 65.7 & 4 & 1.4 & & $40-60$ & $\mathrm{AF}$ & A & 3 \\
\hline 15R-4, 115 & 449.25 & $4 C$ & $\mathrm{M}$ & 73.04 & 86.2 & 4 & 1.0 & & $350-450$ & $\mathrm{TH}$ & B & 5 \\
\hline 16R-1, 22 & 453.42 & 5 & $\mathrm{P}$ & 6.76 & 62.2 & 3 & 1.3 & $\mathrm{AR}$ & $35-45$ & $\mathrm{AF}$ & B & 3 \\
\hline $16 \mathrm{R}-1,38$ & 453.58 & 5 & $\mathrm{P}$ & 7.14 & 41.7 & 4 & 2.2 & AR & $40-60$ & $\mathrm{AF}$ & B & 2 \\
\hline 16R-1, 81 & 454.01 & 5 & $\mathrm{P}$ & 14.46 & 66.5 & 4 & 6.0 & & $425-500$ & $\mathrm{TH}$ & A & 3 \\
\hline 16R-1, 100 & 454.20 & 5 & $P$ & 3.91 & 32.9 & 3 & 3.4 & $\mathrm{AR}$ & $45-60$ & $\mathrm{AF}$ & A & 2 \\
\hline 16R-1, 107 & 454.27 & 5 & $\mathrm{P}$ & 3.90 & 62.7 & 5 & 2.0 & & $20-40$ & $\mathrm{AF}$ & B & 3 \\
\hline 16R-1, 144 & 454.64 & 5 & $\mathrm{P}$ & 2.23 & 44.4 & 4 & 7.5 & PP & $30-45$ & $\mathrm{AF}$ & C & 2 \\
\hline 17R-1, 32 & 461.72 & 5 & $\mathrm{P}$ & 3.19 & 48.3 & 5 & 8.9 & & $400-500$ & $\mathrm{TH}$ & A & 2 \\
\hline $17 \mathrm{R}-1,58$ & 461.98 & 5 & $\mathrm{P}$ & 5.36 & -6.1 & 4 & 2.0 & AR & $40-60$ & $\mathrm{AF}$ & A & 1 \\
\hline 17R-1, 73 & 462.13 & 5 & $\mathrm{P}$ & 7.47 & 75.6 & 4 & 19.3 & PP & $30-45$ & $\mathrm{AF}$ & B & 4 \\
\hline 17R-1, 76 & 462.16 & 5 & $\mathrm{P}$ & 7.26 & 34.5 & 6 & 5.9 & & $375-500$ & $\mathrm{TH}$ & A & 2 \\
\hline $18 \mathrm{R}-1,34$ & 471.34 & 6 & $M$ & 20.80 & 23.7 & 5 & 11.9 & & $400-500$ & $\mathrm{TH}$ & A & 2 \\
\hline $18 R-1,53$ & 471.53 & 6 & $M$ & 5.43 & 7.5 & 4 & 3.5 & AR & $30-50$ & $\mathrm{AF}$ & C & 2 \\
\hline $18 \mathrm{R}-1,55$ & 471.55 & 6 & $\mathrm{M}$ & 8.23 & -17.4 & 3 & 5.0 & & $460-540$ & $\mathrm{TH}$ & A & 5 \\
\hline $18 R-1,65$ & 471.65 & 6 & $M$ & 12.83 & -25.1 & 4 & 2.0 & & $425-500$ & $\mathrm{TH}$ & A & 1 \\
\hline 18R-1, 96 & 471.96 & 6 & $\mathrm{M}$ & 25.51 & & & & PP & & $\mathrm{AF}$ & D & \\
\hline $18 \mathrm{R}-2,13$ & 472.43 & 6 & M & 17.07 & -36.5 & 5 & 1.6 & PP & $20-40$ & $\mathrm{AF}$ & A & 1 \\
\hline $18 R-2,24$ & 472.54 & 6 & $\mathrm{M}$ & 10.28 & 33.9 & 4 & 6.6 & AR & $35-50$ & $\mathrm{AF}$ & B & 2 \\
\hline $18 R-2,32$ & 472.62 & 6 & $\mathrm{M}$ & 30.02 & 67.7 & 3 & 7.1 & & $45-60$ & $\mathrm{AF}$ & $\mathrm{D}$ & 1 \\
\hline $18 \mathrm{R}-2,50$ & 472.70 & 6 & $M$ & 24.14 & -28.2 & 2 & 4.3 & & $420-460$ & $\mathrm{TH}$ & $\mathrm{B}^{\dagger}$ & 5 \\
\hline $18 \mathrm{R}-2,78$ & 473.08 & 6 & $\mathrm{M}$ & 15.11 & & & & PP & & $\mathrm{AF}$ & $\mathrm{D}$ & \\
\hline 18R-2, 102 & 473.32 & 6 & $M$ & 15.69 & 66.60 & 6 & 10.4 & & $300-450$ & $\mathrm{TH}$ & C & 3 \\
\hline $18 \mathrm{R}-2,108$ & 473.38 & 6 & $M$ & 21.42 & & & & & & $\mathrm{AF}$ & $\mathrm{D}$ & \\
\hline $18 \mathrm{R}-3,25$ & 473.81 & 6 & & 43.83 & 59.20 & 5 & 4.3 & & $300-420$ & $\mathrm{TH}$ & B & 3 \\
\hline $18 R-3,44$ & 474.00 & 6 & $\mathrm{M}$ & 26.67 & & & & & & $\mathrm{TH}$ & $\mathrm{D}$ & \\
\hline $18 \mathrm{R}-3,58$ & 474.14 & 6 & $\mathrm{M}$ & 29.42 & 60.50 & 3 & 8.1 & & $500-540$ & $\mathrm{TH}$ & B & 3 \\
\hline 18R-3, 112 & 474.68 & 6 & $\mathrm{M}$ & 26.04 & & & & & & $\mathrm{AF}$ & $\mathrm{D}$ & \\
\hline 18R-4, 21 & 475.09 & 6 & $\mathrm{M}$ & 19.57 & 41.7 & 3 & 3.2 & & $475-525$ & $\mathrm{TH}$ & A & 2 \\
\hline $18 R-4,65$ & 475.53 & 6 & M & 9.78 & 30.7 & 4 & 7.9 & $\mathrm{AR}$ & $30-45$ & $\mathrm{AF}$ & $\mathrm{C}$ & 2 \\
\hline 18R-4, 71 & 475.59 & 6 & $\mathrm{M}$ & 16.16 & & & & PP & & $\mathrm{AF}$ & $\mathrm{D}$ & \\
\hline $18 R-4,84$ & 475.72 & 6 & $\mathrm{M}$ & 4.18 & -4.4 & 3 & 7.2 & & $450-500$ & $\mathrm{TH}$ & $\mathrm{C}$ & 5 \\
\hline $18 \mathrm{R}-4,84$ & 475.72 & 6 & $\mathrm{M}$ & 5.83 & -16.0 & 3 & 10.5 & AR & 40-60 & $\mathrm{AF}$ & A & 1 \\
\hline 18R-4, 112 & 476.00 & $7 A$ & $\mathrm{P}$ & 1.93 & -15.5 & 5 & 4.5 & & $420-540$ & $\mathrm{TH}$ & A & 5 \\
\hline 18R-4, 115 & 476.03 & $7 A$ & $\mathrm{P}$ & 4.06 & & & & PP & & $\mathrm{AF}$ & $\mathrm{D}$ & \\
\hline $18 R-5,5$ & 476.38 & $7 A$ & $\mathrm{P}$ & 6.08 & 23.1 & 5 & 5.4 & & $35-60$ & $\mathrm{AF}$ & B & 2 \\
\hline 19R-1, 21 & 476.31 & $7 A$ & $\mathrm{P}$ & 8.88 & -11.6 & 5 & 0.5 & AR & $35-60$ & $\mathrm{AF}$ & A & 1 \\
\hline 19R-1, 32 & 476.42 & $7 \mathrm{~A}$ & $\mathrm{P}$ & 4.99 & -2.5 & 4 & 6.8 & & $40-60$ & $\mathrm{AF}$ & B & 5 \\
\hline 19R-1, 35 & 476.45 & $7 \mathrm{~A}$ & $\mathrm{P}$ & 2.78 & 6.9 & 3 & 2.7 & & $450-500$ & $\mathrm{TH}$ & A & 2 \\
\hline 19R-1, 47 & 476.57 & $7 \mathrm{~A}$ & $\mathrm{P}$ & 4.80 & 45.6 & 3 & 0.2 & AR & $30-40$ & $\mathrm{AF}$ & B & 2 \\
\hline 19R-1, 51 & 476.61 & $7 \mathrm{~A}$ & $\mathrm{P}$ & 2.67 & 57.5 & 4 & 1.9 & & $25-40$ & $\mathrm{AF}$ & B & 3 \\
\hline 19R-1, 105 & 477.15 & $7 A$ & $\mathrm{P}$ & 9.12 & & & & & & $\mathrm{AF}$ & $\mathrm{D}$ & \\
\hline 19R-2, 28 & 477.85 & $7 A$ & $\mathrm{P}$ & 6.75 & 25.0 & 4 & 15.6 & & $400-475$ & $\mathrm{TH}$ & A & 2 \\
\hline $19 R-2,58$ & 478.15 & $7 A$ & $\mathrm{P}$ & 4.49 & -29.3 & 3 & 0.5 & AR & $35-45$ & $\mathrm{AF}$ & C & 1 \\
\hline $20 \mathrm{R}-1,23$ & 480.83 & $7 A$ & $\mathrm{P}$ & 5.77 & 32.7 & 3 & 1.6 & AR & $30-40$ & $\mathrm{AF}$ & C & 2 \\
\hline $20 \mathrm{R}-1,47$ & 481.07 & $7 \mathrm{~A}$ & $\mathrm{P}$ & 12.78 & -25.2 & 4 & 3.8 & & $425-500$ & $\mathrm{TH}$ & A & 1 \\
\hline 20R-1, 71 & 481.31 & $7 A$ & $\mathrm{P}$ & 4.42 & 36.5 & 4 & 1.0 & $A R$ & $40-60$ & $\mathrm{AF}$ & A & 2 \\
\hline 20R-1, 72 & 481.32 & $7 A$ & $\mathrm{P}$ & 8.34 & & & & & & $\mathrm{AF}$ & D & 2 \\
\hline 20R-1, 117 & 481.77 & $7 A$ & $\mathrm{P}$ & 5.15 & 43.4 & 3 & 0.6 & AR & $30-40$ & $\mathrm{AF}$ & C & 2 \\
\hline $21 \mathrm{R}-1,60$ & 490.08 & $7 \mathrm{~A}$ & $\mathrm{P}$ & 12.31 & 32.6 & 5 & 1.8 & & $25-45$ & $\mathrm{AF}$ & A & 2 \\
\hline 21R-1, 104 & 491.24 & $7 \mathrm{~A}$ & $\mathrm{P}$ & 29.82 & 45.3 & 4 & 2.7 & & $450-525$ & $\mathrm{TH}$ & A & 2 \\
\hline $21 \mathrm{R}-2,14$ & 491.70 & $7 \mathrm{~A}$ & $\mathrm{P}$ & 6.50 & 64.5 & 3 & 5.1 & & $475-525$ & $\mathrm{TH}$ & A & 3 \\
\hline $21 \mathrm{R}-2,30$ & 491.86 & $7 B$ & $\mathrm{P}$ & 5.36 & $-61.6^{*}$ & 3 & 2.1 & $\mathrm{AR}$ & 45-60 & $\mathrm{AF}$ & B & 3 \\
\hline $21 \mathrm{R}-2,30$ & 491.86 & $7 B$ & $\mathrm{P}$ & 3.47 & 15.5 & 4 & 3.8 & & $370-500$ & $\mathrm{TH}$ & B & 2 \\
\hline $21 R-2,62$ & 492.18 & $7 B$ & $\mathrm{P}$ & 1.77 & 18.00 & 4 & 2.9 & $A R$ & $40-60$ & $\mathrm{AF}$ & C & 2 \\
\hline $21 \mathrm{R}-2,99$ & 492.55 & $7 B$ & $P$ & 5.13 & & & & & & $\mathrm{TH}$ & $\mathrm{D}$ & \\
\hline 21R-2, 136 & 492.92 & $7 B$ & $\mathrm{P}$ & 3.15 & 49.1 & 3 & 0.9 & $A R$ & $35-45$ & $\mathrm{AF}$ & A & 2 \\
\hline $21 \mathrm{R}-2,138$ & 492.94 & $7 B$ & $P$ & 2.56 & -21.6 & 4 & 6.6 & & $460-540$ & $\mathrm{TH}$ & A & 5 \\
\hline $21 \mathrm{R}-3,46$ & 493.45 & $7 B$ & $P$ & 5.51 & & & & & & $\mathrm{AF}$ & B & 2 \\
\hline $21 \mathrm{R}-3,55$ & 493.54 & $7 B$ & $\mathrm{P}$ & 3.34 & 56.1 & 4 & 1.1 & AR & $30-45$ & $\mathrm{AF}$ & B & 3 \\
\hline $21 \mathrm{R}-3,118$ & 494.17 & $7 B$ & $\mathrm{P}$ & 3.12 & 11.5 & 4 & 6.6 & & $40-60$ & $\mathrm{AF}$ & B & 2 \\
\hline $21 R-4,3$ & 494.52 & $7 B$ & $\mathrm{P}$ & 8.00 & -16.6 & 3 & 7.2 & & $450-500$ & $\mathrm{TH}$ & A & 5 \\
\hline $21 R-4,49$ & 494.98 & $7 B$ & $\mathrm{P}$ & 5.05 & 60.5 & 4 & 1.3 & $A R$ & $40-60$ & $\mathrm{AF}$ & A & 3 \\
\hline 21R-4, 71 & 495.20 & $7 B$ & $\mathrm{P}$ & 31.86 & 52.9 & 5 & 4.1 & & $425-525$ & $\mathrm{TH}$ & A & 3 \\
\hline
\end{tabular}


Table T1 (continued). (Continued on next page.)

\begin{tabular}{|c|c|c|c|c|c|c|c|c|c|c|c|c|}
\hline \multirow{2}{*}{$\begin{array}{l}\text { Core, section, } \\
\text { interval }(\mathrm{cm})\end{array}$} & \multirow{2}{*}{$\begin{array}{l}\text { Depth } \\
\text { (mbsf) }\end{array}$} & \multirow[b]{2}{*}{ Unit } & \multirow{2}{*}{$\begin{array}{l}\text { Unit } \\
\text { type }\end{array}$} & \multirow{2}{*}{$\begin{array}{l}\text { NRM } \\
(\mathrm{A} / \mathrm{m})\end{array}$} & \multirow{2}{*}{$\begin{array}{c}\text { Inc. } \\
\left({ }^{\circ}\right)\end{array}$} & \multirow[b]{2}{*}{$N$} & & & Den & magnetizatic & & ChRM \\
\hline & & & & & & & MAD & type & Steps & Treatment & Quality & type \\
\hline $22 \mathrm{R}-1,23$ & 495.63 & $7 B$ & $\mathrm{P}$ & 15.86 & $-32.6^{\star}$ & 4 & 3.4 & & $40-60$ & $\mathrm{AF}$ & A & 2 \\
\hline $22 \mathrm{R}-1,53$ & 495.93 & $7 B$ & $P$ & 3.55 & 49.9 & 5 & 0.7 & & $35-60$ & $\mathrm{AF}$ & A & 2 \\
\hline $22 \mathrm{R}-1,96$ & 496.36 & $7 B$ & $P$ & 11.16 & & & & AR & & $\mathrm{AF}$ & $\mathrm{D}$ & \\
\hline 22R-1, 107 & 496.47 & $7 B$ & $P$ & 5.65 & & & & AR & & $\mathrm{AF}$ & $\mathrm{D}$ & \\
\hline 22R-1, 131 & 496.71 & $7 B$ & $\mathrm{P}$ & 11.74 & 57.6 & 4 & 5.2 & & $425-500$ & $\mathrm{TH}$ & B & 2 \\
\hline $22 \mathrm{R}-2,5$ & 496.88 & $7 B$ & $\mathrm{P}$ & 4.60 & 58.4 & 5 & 0.7 & $A R$ & $35-60$ & $\mathrm{AF}$ & A & 3 \\
\hline $22 \mathrm{R}-2,26$ & 497.09 & $7 B$ & $\mathrm{P}$ & 10.10 & 64.1 & 3 & 9.7 & & $475-525$ & $\mathrm{TH}$ & A & 3 \\
\hline $22 \mathrm{R}-2,48$ & 497.31 & $7 B$ & $\mathrm{P}$ & 11.16 & 12.7 & 4 & 1.0 & $A R$ & $30-45$ & $\mathrm{AF}$ & A & 2 \\
\hline $22 \mathrm{R}-2,60$ & 497.43 & $7 B$ & $\mathrm{P}$ & 7.95 & 50.8 & 5 & 2.0 & & $30-50$ & $\mathrm{AF}$ & A & 3 \\
\hline $23 \mathrm{R}-1,28$ & 500.18 & $7 B$ & $P$ & 10.81 & 50.8 & 5 & 2.2 & & $380-540$ & $\mathrm{TH}$ & A & 3 \\
\hline $23 \mathrm{R}-1,29$ & 500.19 & $7 B$ & $P$ & 7.11 & 46.9 & 4 & 1.9 & AR & $40-60$ & $\mathrm{AF}$ & A & 2 \\
\hline $23 \mathrm{R}-1,50$ & 500.40 & $7 B$ & $\mathrm{P}$ & 2.70 & -31.5 & 5 & 14.1 & & $425-525$ & $\mathrm{TH}$ & A & 1 \\
\hline $23 R-1,66$ & 500.56 & $7 B$ & $P$ & 4.81 & 37.6 & 3 & 1.1 & AR & 45-60 & $\mathrm{AF}$ & B & 2 \\
\hline 23R-1, 93 & 500.83 & $7 B$ & $P$ & 4.48 & & & & & & $\mathrm{TH}$ & & \\
\hline 23R-1, 131 & 501.20 & $7 B$ & $\mathrm{P}$ & 5.81 & 50.4 & 4 & 2.5 & AR & $40-60$ & $\mathrm{AF}$ & B & 3 \\
\hline $23 \mathrm{R}-2,10$ & 501.46 & $7 \mathrm{~B}$ & $P$ & 10.47 & & & & & & $\mathrm{AF}$ & C & \\
\hline $23 \mathrm{R}-2,30$ & 501.66 & $7 B$ & $\mathrm{P}$ & 3.56 & 46.5 & 4 & 8.4 & & $460-540$ & $\mathrm{TH}$ & A & 2 \\
\hline $23 \mathrm{R}-2,46$ & 501.82 & $7 B$ & $\mathrm{P}$ & 5.62 & 50.6 & 3 & 4.3 & $A R$ & $45-60$ & $\mathrm{AF}$ & B & 3 \\
\hline $23 R-2,89$ & 502.25 & $7 \mathrm{~B}$ & $\mathrm{P}$ & 4.61 & -18.8 & 4 & 3.2 & & $460-540$ & $\mathrm{TH}$ & A & 1 \\
\hline $23 \mathrm{R}-2,141$ & 502.77 & $7 B$ & $\mathrm{P}$ & 7.64 & & & & & & $\mathrm{AF}$ & C & \\
\hline $23 R-3,8$ & 502.94 & $7 B$ & $\mathrm{P}$ & 17.42 & 58.5 & 4 & 2.9 & & $425-500$ & $\mathrm{TH}$ & A & 3 \\
\hline $23 \mathrm{R}-3,24$ & 503.10 & $7 B$ & $\mathrm{P}$ & 8.47 & 32.5 & 4 & 1.1 & AR & $30-45$ & $\mathrm{AF}$ & B & 2 \\
\hline $24 \mathrm{R}-1,10$ & 506.00 & $7 B$ & $P$ & 3.68 & -23.2 & 3 & 3.9 & & $400-500$ & $\mathrm{TH}$ & B & 5 \\
\hline $24 \mathrm{R}-1,13$ & 506.03 & $7 B$ & $\mathrm{P}$ & 3.72 & 9.9 & 4 & 0.6 & $A R$ & $35-50$ & $\mathrm{AF}$ & B & 2 \\
\hline $24 \mathrm{R}-1,34$ & 506.24 & $7 B$ & $\mathrm{P}$ & 7.14 & -11.8 & 6 & 11.1 & & $375-525$ & $\mathrm{TH}$ & $\mathrm{C}$ & 1 \\
\hline $24 \mathrm{R}-1,55$ & 506.45 & $7 B$ & $P$ & 3.61 & 23.3 & 4 & 7.3 & & $460-540$ & $\mathrm{TH}$ & A & 5 \\
\hline $24 \mathrm{R}-1,59$ & 506.49 & $7 B$ & $\mathrm{P}$ & 3.73 & 22.0 & 3 & 1.0 & AR & $35-45$ & $\mathrm{AF}$ & C & 2 \\
\hline $24 \mathrm{R}-1,89$ & 506.79 & $7 B$ & $\mathrm{P}$ & 5.19 & 32.0 & 4 & 2.4 & AR & $35-50$ & $\mathrm{AF}$ & C & 2 \\
\hline 24R-1, 103 & 506.93 & $7 \mathrm{~B}$ & $\mathrm{P}$ & 10.73 & 53.1 & 4 & 1.1 & AR & $35-50$ & $\mathrm{AF}$ & A & 3 \\
\hline $24 \mathrm{R}-1,126$ & 507.16 & $7 B$ & $\mathrm{P}$ & 30.43 & 53.7 & 6 & 10.2 & & $400-525$ & $\mathrm{TH}$ & A & 3 \\
\hline $24 \mathrm{R}-2,8$ & 507.30 & $7 B$ & $\mathrm{P}$ & 3.00 & 35.4 & 3 & 2.6 & $A R$ & $35-45$ & $\mathrm{AF}$ & A & 2 \\
\hline $24 \mathrm{R}-2,9$ & 507.31 & $7 B$ & $\mathrm{P}$ & 32.91 & 51.1 & 4 & 17.6 & & $400-500$ & $\mathrm{TH}$ & B & 4 \\
\hline $24 \mathrm{R}-2,37$ & 507.59 & $7 B$ & $\mathrm{P}$ & 32.83 & 41.6 & 3 & 2.3 & & $475-525$ & $\mathrm{TH}$ & A & 2 \\
\hline $25 \mathrm{R}-1,11$ & 509.61 & $7 \mathrm{~B}$ & $\mathrm{P}$ & 5.80 & 66.6 & 4 & 0.7 & AR & $35-50$ & $\mathrm{AF}$ & B & 3 \\
\hline $25 \mathrm{R}-1,42$ & 509.92 & $7 B$ & $\mathrm{P}$ & 3.21 & -51.9 & 4 & 0.9 & & $25-40$ & $\mathrm{AF}$ & B & 1 \\
\hline $25 \mathrm{R}-1,54$ & 510.04 & $7 B$ & $P$ & 8.14 & 61.0 & 3 & 11.4 & & $475-525$ & $\mathrm{TH}$ & B & \\
\hline $25 \mathrm{R}-1,75$ & 510.25 & $7 B$ & $\mathrm{P}$ & 4.46 & 55.8 & 4 & 2.5 & $A R$ & $35-50$ & $\mathrm{AF}$ & A & 3 \\
\hline $25 \mathrm{R}-1,110$ & 510.60 & $7 B$ & $P$ & 4.46 & 52.8 & 3 & 1.3 & AR & $35-45$ & $\mathrm{AF}$ & C & 3 \\
\hline $25 \mathrm{R}-1,132$ & 510.82 & $7 B$ & $\mathrm{P}$ & 15.32 & 74.2 & 5 & 3.9 & & $400-500$ & $\mathrm{TH}$ & A & 3 \\
\hline $25 \mathrm{R}-2,6$ & 510.97 & $7 \mathrm{~B}$ & $\mathrm{P}$ & 9.70 & 40.3 & 3 & 7.3 & & $475-525$ & $\mathrm{TH}$ & A & 2 \\
\hline $25 \mathrm{R}-2,55$ & 511.46 & $7 B$ & $\mathrm{P}$ & 3.90 & 43.6 & 3 & 1.5 & AR & $35-45$ & $\mathrm{AF}$ & B & 2 \\
\hline $25 \mathrm{R}-2,76$ & 511.67 & 7B & $\mathrm{P}$ & 6.08 & 50.9 & 3 & 0.8 & $A R$ & $35-45$ & $\mathrm{AF}$ & C & 3 \\
\hline $25 \mathrm{R}-2,97$ & 511.88 & 7C & $\mathrm{P}$ & 11.35 & -5.5 & 3 & 0.9 & & $35-45$ & $\mathrm{AF}$ & B & 1 \\
\hline $26 \mathrm{R}-1,37$ & 515.87 & 7C & $\mathrm{P}$ & 4.36 & -69.4 & 5 & 7.9 & & $450-500$ & $\mathrm{TH}$ & A & 5 \\
\hline $26 \mathrm{R}-1,37$ & 515.87 & $7 C$ & $\mathrm{P}$ & 3.13 & 51.3 & 4 & 1.2 & $A R$ & $20-40$ & $\mathrm{AF}$ & A & 3 \\
\hline $27 \mathrm{R}-1,66$ & 519.66 & $7 C$ & $\mathrm{P}$ & 4.49 & 66.8 & 4 & 0.6 & AR & $30-45$ & $\mathrm{AF}$ & A & 3 \\
\hline $27 R-1,68$ & 519.68 & 7C & $\mathrm{P}$ & 10.26 & & & & & & $\mathrm{AF}$ & C & \\
\hline 27R-1, 84 & 519.84 & $7 C$ & $\mathrm{P}$ & 15.91 & 54.1 & 3 & 1.5 & & $475-525$ & $\mathrm{TH}$ & A & 3 \\
\hline 27R-1, 136 & 520.36 & $7 C$ & $P$ & 6.75 & $-65.1^{*}$ & 4 & 0.9 & AR & $35-50$ & $\mathrm{AF}$ & B & 3 \\
\hline $27 \mathrm{R}-2,4$ & 520.54 & $7 C$ & $\mathrm{P}$ & 5.98 & 62.9 & 3 & 1.6 & $A R$ & $35-45$ & $\mathrm{AF}$ & C & 3 \\
\hline $27 \mathrm{R}-2,13$ & 520.63 & $7 C$ & $\mathrm{P}$ & 6.12 & 69.0 & 3 & 0.5 & AR & $30-40$ & $\mathrm{AF}$ & A & 3 \\
\hline $27 \mathrm{R}-2,26$ & 520.76 & 7C & $\mathrm{P}$ & 17.10 & 68.4 & 4 & 12.5 & & $425-500$ & $\mathrm{TH}$ & A & 3 \\
\hline 28R-1, 19 & 528.79 & $7 C$ & $P$ & 8.40 & & & & & & $\mathrm{TH}$ & C & \\
\hline $28 \mathrm{R}-1,46$ & 529.06 & 7C & $\mathrm{P}$ & 16.31 & 44.2 & 3 & 5.9 & & $475-525$ & $\mathrm{TH}$ & A & 2 \\
\hline $28 \mathrm{R}-1,49$ & 529.09 & $7 C$ & $\mathrm{P}$ & 6.33 & 66.3 & 3 & 0.7 & $A R$ & $30-40$ & $\mathrm{AF}$ & C & 3 \\
\hline $30 \mathrm{R}-1,47$ & 535.67 & 7C & $\mathrm{P}$ & 8.61 & 48.7 & 4 & 2.3 & $A R$ & $40-60$ & $\mathrm{AF}$ & B & 2 \\
\hline $30 R-1,63$ & 535.83 & 7C & $\mathrm{P}$ & 4.66 & 63.2 & 3 & 19.1 & & $500-550$ & $\mathrm{TH}$ & C & 3 \\
\hline $30 R-1,98$ & 536.18 & $7 C$ & $\mathrm{P}$ & 5.35 & 47.6 & 4 & 0.7 & $A R$ & $35-50$ & $\mathrm{AF}$ & A & 2 \\
\hline 30R-1, 99 & 536.19 & $7 C$ & $\mathrm{P}$ & 4.22 & -61.0 & 3 & 0.9 & & $450-525$ & $\mathrm{TH}$ & A & 5 \\
\hline $30 \mathrm{R}-1,106$ & 536.26 & $7 C$ & $\mathrm{P}$ & 3.13 & -46.2 & 3 & 5.1 & & $500-550$ & $\mathrm{TH}$ & A & 5 \\
\hline 30R-1, 116 & 536.36 & $7 C$ & $\mathrm{P}$ & 7.59 & -54.7 & 3 & 2.3 & $A R$ & $45-60$ & $\mathrm{AF}$ & A & 1 \\
\hline $31 \mathrm{R}-1,49$ & 544.89 & $7 C$ & $P$ & 3.18 & 52.9 & 3 & 7.2 & AR & $45-60$ & $\mathrm{AF}$ & C & 3 \\
\hline $31 \mathrm{R}-1,51$ & 544.91 & $7 C$ & $\mathrm{P}$ & 3.37 & -43.7 & 3 & 9.2 & & $500-550$ & $\mathrm{TH}$ & A & 1 \\
\hline $32 \mathrm{R}-1,19$ & 550.19 & 7C & $P$ & 2.64 & -8.3 & 4 & 1.4 & AR & $30-45$ & $\mathrm{AF}$ & B & 1 \\
\hline $32 \mathrm{R}-1,36$ & 550.36 & 7C & $\mathrm{P}$ & 11.36 & 21.8 & 4 & 3.9 & & $40-60$ & $\mathrm{AF}$ & A & 2 \\
\hline $32 \mathrm{R}-1,81$ & 550.81 & $7 C$ & $\mathrm{P}$ & 4.99 & 39.9 & 3 & 2.4 & & $475-550$ & $\mathrm{TH}$ & A & 2 \\
\hline $32 \mathrm{R}-1,119$ & 551.19 & 7C & $\mathrm{P}$ & 3.66 & 55.4 & 3 & 3.2 & PP & 20-30 & $\mathrm{AF}$ & $\mathrm{C}$ & 4 \\
\hline $32 \mathrm{R}-1,129$ & 551.29 & $7 C$ & $\mathrm{P}$ & 18.58 & 22.0 & 3 & 0.6 & $A R$ & $35-45$ & $\mathrm{AF}$ & C & 2 \\
\hline $32 \mathrm{R}-2,18$ & 551.62 & $7 C$ & $\mathrm{P}$ & 5.28 & & & & & & $\mathrm{AF}$ & C & \\
\hline
\end{tabular}


Table T1 (continued).

\begin{tabular}{|c|c|c|c|c|c|c|c|c|c|c|c|c|}
\hline \multirow{2}{*}{$\begin{array}{l}\text { Core, section, } \\
\text { interval }(\mathrm{cm})\end{array}$} & \multirow{2}{*}{$\begin{array}{l}\text { Depth } \\
\text { (mbsf) }\end{array}$} & \multirow[b]{2}{*}{ Unit } & \multirow{2}{*}{$\begin{array}{l}\text { Unit } \\
\text { type }\end{array}$} & \multirow{2}{*}{$\begin{array}{l}\text { NRM } \\
(\mathrm{A} / \mathrm{m})\end{array}$} & \multirow{2}{*}{$\begin{array}{c}\text { Inc. } \\
\left({ }^{\circ}\right)\end{array}$} & \multirow[b]{2}{*}{$N$} & \multirow[b]{2}{*}{ MAD } & \multirow{2}{*}{$\begin{array}{c}\text { Sample } \\
\text { type }\end{array}$} & \multicolumn{3}{|c|}{ Demagnetization } & \multirow{2}{*}{$\begin{array}{l}\text { ChRM } \\
\text { type }\end{array}$} \\
\hline & & & & & & & & & Steps & Treatment & Quality & \\
\hline $32 R-2,54$ & 551.98 & 7C & $\mathrm{P}$ & 5.84 & 55.8 & 3 & 0.2 & $A R$ & $40-50$ & $\mathrm{AF}$ & B & 3 \\
\hline $32 \mathrm{R}-2,83$ & 552.27 & $7 C$ & $P$ & 15.50 & -30.2 & 3 & 1.9 & $A R$ & $40-50$ & $\mathrm{AF}$ & B & 1 \\
\hline $32 \mathrm{R}-2,105$ & 552.49 & 7C & $P$ & 8.71 & 60.0 & 3 & 43.1 & & $500-550$ & $\mathrm{TH}$ & B & 3 \\
\hline $32 \mathrm{R}-3,4$ & 552.98 & 7C & $P$ & 10.71 & -13.0 & 3 & 14.8 & & $450-525$ & $\mathrm{TH}$ & $\mathrm{C}$ & 1 \\
\hline $32 \mathrm{R}-3,19$ & 553.13 & 7C & $P$ & 5.09 & -2.5 & 4 & 2.1 & $A R$ & $35-50$ & $\mathrm{AF}$ & B & 1 \\
\hline $32 \mathrm{R}-3,33$ & 553.27 & $7 C$ & $\mathrm{P}$ & 7.23 & -2.9 & 3 & 10.9 & & $500-550$ & $\mathrm{TH}$ & A & 1 \\
\hline $33 R-1,67$ & 554.67 & 7C & $\mathrm{P}$ & 2.40 & 64.0 & 3 & 7.4 & & $500-550$ & $\mathrm{TH}$ & $A$ & 3 \\
\hline $33 \mathrm{R}-1,83$ & 554.83 & $7 C$ & $\mathrm{P}$ & 6.41 & 58.9 & 3 & 0.6 & $A R$ & $30-40$ & $\mathrm{AF}$ & B & \\
\hline $33 \mathrm{R}-1,110$ & 555.10 & $7 C$ & $\mathrm{P}$ & 15.56 & & & & & & $\mathrm{AF}$ & $\mathrm{C}$ & \\
\hline $33 \mathrm{R}-1,132$ & 555.32 & 7C & $P$ & 3.53 & 43.4 & 4 & 1.7 & AR & $30-45$ & $\mathrm{AF}$ & B & 2 \\
\hline $33 \mathrm{R}-2,7$ & 555.57 & 7C & $P$ & 5.64 & 34.9 & 3 & 1.2 & AR & $40-50$ & $\mathrm{AF}$ & $A$ & 2 \\
\hline $33 \mathrm{R}-2,19$ & 555.69 & 7C & $P$ & 6.16 & -36.1 & 4 & 9.7 & & $475-550$ & $\mathrm{TH}$ & $A$ & 1 \\
\hline $33 \mathrm{R}-2,40$ & 555.90 & $7 C$ & $\mathrm{P}$ & 2.17 & 78.5 & 3 & 1.4 & AR & $30-40$ & $\mathrm{AF}$ & C & 3 \\
\hline $33 R-2,105$ & 556.55 & $7 C$ & $P$ & 3.11 & 37.7 & 4 & 1.6 & $A R$ & $40-60$ & $\mathrm{AF}$ & B & 2 \\
\hline $33 R-2,124$ & 556.74 & 7C & $P$ & 5.44 & 23.5 & 3 & 1.5 & & $500-550$ & $\mathrm{TH}$ & $A$ & 2 \\
\hline $34 \mathrm{R}-1,3$ & 560.63 & $7 C$ & $P$ & 6.33 & 36.6 & 5 & 14.2 & & $425-550$ & $\mathrm{TH}$ & $A$ & 2 \\
\hline $34 \mathrm{R}-1,28$ & 560.88 & 7C & $P$ & 7.96 & -38.8 & 4 & 1.1 & AR & $35-50$ & $\mathrm{AF}$ & C & 1 \\
\hline $34 \mathrm{R}-1,49$ & 561.09 & 7C & $P$ & 3.95 & 32.7 & 3 & 12.6 & & $500-550$ & $\mathrm{TH}$ & A & 2 \\
\hline $34 \mathrm{R}-1,80$ & 561.40 & $7 C$ & $\mathrm{P}$ & 4.57 & 11.1 & 3 & 0.7 & AR & $35-45$ & $\mathrm{AF}$ & B & 2 \\
\hline $34 \mathrm{R}-2,26$ & 562.36 & $7 C$ & $P$ & 4.16 & -46.5 & 4 & 4.5 & & $450-525$ & $\mathrm{TH}$ & A & 1 \\
\hline $34 \mathrm{R}-2,35$ & 562.45 & 7C & $P$ & 4.44 & 51.2 & 3 & 0.9 & AR & $40-50$ & $\mathrm{AF}$ & B & 3 \\
\hline $34 \mathrm{R}-2,87$ & 562.97 & 7C & $\mathrm{P}$ & 4.64 & -72.5 & 3 & 1.5 & AR & $35-45$ & $\mathrm{AF}$ & A & 1 \\
\hline $34 \mathrm{R}-2,90$ & 563.00 & 7C & $P$ & 13.15 & -65.4 & 3 & 3.7 & & $500-540$ & $\mathrm{TH}$ & B & 3 \\
\hline $34 \mathrm{R}-2,98$ & 563.08 & $7 C$ & $\mathrm{P}$ & 7.85 & -46.3 & 4 & 2.3 & $A R$ & $30-45$ & $\mathrm{AF}$ & $A$ & 3 \\
\hline $34 \mathrm{R}-2,119$ & 563.29 & $7 C$ & $\mathrm{P}$ & 9.10 & 11.5 & 4 & 5.3 & & $475-550$ & $\mathrm{TH}$ & B & 2 \\
\hline $35 \mathrm{R}-1,16$ & 563.76 & $7 C$ & $P$ & 3.05 & -0.6 & 4 & 4.3 & & $475-550$ & $\mathrm{TH}$ & B & 1 \\
\hline $35 \mathrm{R}-1,27$ & 563.87 & $7 C$ & $\mathrm{P}$ & 7.61 & -14.2 & 4 & 14.2 & & $450-525$ & $\mathrm{TH}$ & A & 4 \\
\hline $35 \mathrm{R}-1,54$ & 564.14 & $7 C$ & $P$ & 3.17 & & & & $A R$ & & $\mathrm{AF}$ & & \\
\hline $35 \mathrm{R}-1,72$ & 564.32 & 7C & $P$ & 1.88 & -28.2 & 5 & 2.8 & & $350-500$ & $\mathrm{TH}$ & A & 5 \\
\hline $35 \mathrm{R}-1,77$ & 564.37 & 7C & $P$ & 7.17 & 45.2 & 3 & 1.3 & AR & $40-50$ & $\mathrm{AF}$ & $A$ & 2 \\
\hline $35 \mathrm{R}-2,20$ & 565.27 & $8 \mathrm{~A}$ & B & 6.03 & 51.2 & 3 & 1.2 & $A R$ & $35-45$ & $\mathrm{AF}$ & C & 3 \\
\hline $35 \mathrm{R}-2,41$ & 565.48 & $8 \mathrm{~A}$ & B & 5.07 & 38.2 & 4 & 1.3 & $A R$ & $35-50$ & $\mathrm{AF}$ & A & 2 \\
\hline $35 R-2,96$ & 566.03 & $8 \mathrm{~B}$ & $\mathrm{P}$ & 6.04 & 40.4 & 6 & 4.6 & & $300-450$ & $\mathrm{TH}$ & A & 2 \\
\hline $35 \mathrm{R}-2,104$ & 566.11 & $8 B$ & $P$ & 27.67 & 64.2 & 4 & 17.9 & & $450-550$ & $\mathrm{TH}$ & A & 3 \\
\hline $35 R-2,118$ & 566.25 & $8 B$ & $\mathrm{P}$ & 10.46 & 38.8 & 3 & 18.4 & & $475-525$ & $\mathrm{TH}$ & A & 2 \\
\hline $35 \mathrm{R}-2,128$ & 566.35 & $8 B$ & $\mathrm{P}$ & 5.30 & 34.9 & 4 & 2.2 & $A R$ & $40-60$ & $\mathrm{AF}$ & A & 2 \\
\hline $35 \mathrm{R}-2,130$ & 566.37 & $8 B$ & $\mathrm{P}$ & 44.43 & 48.70 & 12.1 & 3 & & $245-270$ & $\mathrm{TH}$ & B & 2 \\
\hline $35 \mathrm{R}-3,12$ & 556.61 & $8 \mathrm{~B}$ & $\mathrm{P}$ & 21.16 & 32.1 & 4 & 17.5 & & $450-525$ & $\mathrm{TH}$ & A & 2 \\
\hline $36 \mathrm{R}-1,12$ & 573.32 & $8 C$ & $P$ & 3.25 & -40.0 & 3 & 1.7 & & $460-540$ & $\mathrm{TH}$ & A & 5 \\
\hline $36 \mathrm{R}-1,30$ & 573.50 & $8 C$ & $\mathrm{P}$ & 16.51 & 66.5 & 4 & 2.3 & $A R$ & $40-60$ & $\mathrm{AF}$ & $\mathrm{C}$ & 3 \\
\hline $36 \mathrm{R}-1,41$ & 573.61 & $8 C$ & $P$ & 7.00 & 34.6 & 3 & 2.7 & AR & $35-45$ & $\mathrm{AF}$ & $\mathrm{C}$ & 2 \\
\hline $36 \mathrm{R}-1,90$ & 574.10 & $8 C$ & $P$ & 3.26 & -52.6 & 5 & 6.3 & & $450-550$ & $\mathrm{TH}$ & $A$ & 1 \\
\hline $36 \mathrm{R}-1,124$ & 574.44 & $8 C$ & $\mathrm{P}$ & 5.74 & 56.4 & 3 & 0.7 & $\mathrm{AR}$ & $35-45$ & $\mathrm{AF}$ & B & 3 \\
\hline $36 \mathrm{R}-1,144$ & 574.64 & $8 C$ & $\mathrm{P}$ & 6.09 & -19.9 & 2 & 7.5 & & $525-550$ & $\mathrm{TH}$ & C & 5 \\
\hline $36 \mathrm{R}-2,8$ & 574.78 & $8 C$ & $\mathrm{P}$ & 4.25 & -50.9 & 3 & 1.4 & & $500-550$ & $\mathrm{TH}$ & A & 5 \\
\hline $36 \mathrm{R}-2,85$ & 575.55 & $8 C$ & $\mathrm{P}$ & 5.43 & 52.8 & 3 & 2.2 & $A R$ & $35-45$ & $\mathrm{AF}$ & B & 3 \\
\hline 36R-2, 101 & 575.71 & $8 C$ & $P$ & 8.19 & 53.9 & 3 & 1.7 & $A R$ & $25-35$ & $\mathrm{AF}$ & C & 3 \\
\hline $36 \mathrm{R}-2,122$ & 575.92 & $8 C$ & $\mathrm{P}$ & 6.63 & -55.3 & 4 & 3.3 & & $475-550$ & $\mathrm{TH}$ & A & 5 \\
\hline $36 \mathrm{R}-3,36$ & 576.53 & $8 C$ & $P$ & 3.76 & -61.9 & 3 & 0.9 & & $500-550$ & $\mathrm{TH}$ & A & 1 \\
\hline $36 \mathrm{R}-3,85$ & 577.02 & $8 C$ & $\mathrm{P}$ & 3.10 & -25.1 & 4 & 9.6 & & $450-550$ & $\mathrm{TH}$ & A & 1 \\
\hline
\end{tabular}

Notes: See the "Site U1301" chapter for igneous unit definitions. NRM = natural remanent magnetization, Inc. = inclination, $N=$ number of measurements used for PCA analysis, MAD = maximum angle of deflection (a measure of the error in PCA analysis), Demagnetization quality $=$ subjective judgement of demagnetization curve consistency ranging from $\mathrm{A}=$ excellent, simple isovectorial decay, to $\mathrm{C}=$ poor, poor consistency, small number of consistent steps, or departure from isovectorial decay, ChRM type = subjective classification of characteristic remanent magnetization direction. $\mathrm{P}=$ pillow basalt, $\mathrm{M}=$ massive flow. $\mathrm{AR}=$ archive-half sample, $\mathrm{PP}=$ physical properties sample. $\mathrm{AF}=$ alternating field, $\mathrm{TH}=$ thermal. ${ }^{*}=$ thought to be inverted samples. $\dagger=$ ChRM inclinations calculated using a PCA solution anchored to the origin. 


\begin{tabular}{|c|c|c|c|c|c|c|c|c|c|c|c|c|c|c|c|c|}
\hline \multirow{3}{*}{$\begin{array}{l}\text { Core, section, } \\
\text { interval }(\mathrm{cm}) \text { : }\end{array}$} & \multicolumn{16}{|c|}{ Hole U1301B } \\
\hline & \multicolumn{2}{|c|}{$7 \mathrm{R}-1,12$} & \multicolumn{2}{|c|}{$7 \mathrm{R}-1,12$} & \multicolumn{2}{|c|}{$15 \mathrm{R}-1,44$} & \multicolumn{2}{|c|}{$15 \mathrm{R}-1,44$} & \multicolumn{2}{|c|}{$15 \mathrm{R}-2,141$} & \multicolumn{2}{|c|}{$15 \mathrm{R}-2,141$} & \multicolumn{2}{|c|}{$15 R-4,135$} & \multicolumn{2}{|c|}{$15 R-4,135$} \\
\hline & $\begin{array}{l}\text { Field } \\
(\mathrm{Oe})\end{array}$ & $\begin{array}{l}\text { Halo } M \\
\text { (emu) }\end{array}$ & $\begin{array}{l}\text { Field } \\
(\mathrm{Oe})\end{array}$ & $\begin{array}{l}\text { Normal } M \\
(\mathrm{emu})\end{array}$ & $\begin{array}{l}\text { Field } \\
(\mathrm{Oe})\end{array}$ & $\begin{array}{l}\text { Halo } M \\
\text { (emu) }\end{array}$ & $\begin{array}{l}\text { Field } \\
(\mathrm{Oe})\end{array}$ & $\begin{array}{l}\text { Normal } M \\
(\mathrm{emu})\end{array}$ & $\begin{array}{l}\text { Field } \\
(\mathrm{Oe})\end{array}$ & $\begin{array}{l}\text { Halo } M \\
\text { (emu) }\end{array}$ & $\begin{array}{l}\text { Field } \\
(\mathrm{Oe})\end{array}$ & $\begin{array}{l}\text { Normal } M \\
(\mathrm{emu})\end{array}$ & $\begin{array}{l}\text { Field } \\
(\mathrm{Oe})\end{array}$ & $\begin{array}{l}\text { Halo } M \\
\text { (emu) }\end{array}$ & $\begin{array}{l}\text { Field } \\
(\mathrm{Oe})\end{array}$ & $\begin{array}{c}\text { Normal } M \\
\text { (emu) }\end{array}$ \\
\hline 1 & 0.8 & $2.08 \mathrm{E}-04$ & 0.9 & $6.68 \mathrm{E}-05$ & 0.9 & $1.34 \mathrm{E}-04$ & 0.9 & $3.59 \mathrm{E}-04$ & 0.9 & $1.45 \mathrm{E}-04$ & 0.8 & $5.33 \mathrm{E}-05$ & 0.9 & $1.82 \mathrm{E}-04$ & 0.9 & 8.17E-05 \\
\hline 2 & 128.0 & $1.99 \mathrm{E}-03$ & 128.0 & $6.78 \mathrm{E}-04$ & 128.1 & 4.44E-03 & 128.0 & $1.18 \mathrm{E}-02$ & 250.4 & $1.04 \mathrm{E}-02$ & 250.5 & $3.37 \mathrm{E}-03$ & 128.0 & $1.65 \mathrm{E}-03$ & 128.1 & $9.10 \mathrm{E}-04$ \\
\hline 3 & 250.3 & $1.10 \mathrm{E}-02$ & 250.5 & $3.06 \mathrm{E}-03$ & 250.5 & $8.80 \mathrm{E}-03$ & 250.4 & $1.88 \mathrm{E}-02$ & 500.1 & $1.90 \mathrm{E}-02$ & 500.1 & $6.29 \mathrm{E}-03$ & 250.5 & $9.87 \mathrm{E}-03$ & 250.5 & $4.45 \mathrm{E}-03$ \\
\hline 4 & 377.5 & $1.96 \mathrm{E}-02$ & 377.5 & $4.36 \mathrm{E}-03$ & 377.6 & $1.06 \mathrm{E}-02$ & 377.7 & $2.10 \mathrm{E}-02$ & 754.5 & $2.16 \mathrm{E}-02$ & 754.5 & $7.05 \mathrm{E}-03$ & 377.7 & $1.79 \mathrm{E}-02$ & 377.7 & $7.20 \mathrm{E}-03$ \\
\hline 5 & 499.9 & $2.40 \mathrm{E}-02$ & 499.9 & $4.78 \mathrm{E}-03$ & 500.0 & $1.14 \mathrm{E}-02$ & 500.0 & $2.18 \mathrm{E}-02$ & $1,003.6$ & $2.26 \mathrm{E}-02$ & $1,003.7$ & $7.30 \mathrm{E}-03$ & 500.0 & $2.25 \mathrm{E}-02$ & 500.1 & $8.59 \mathrm{E}-03$ \\
\hline 6 & 626.9 & $2.60 \mathrm{E}-02$ & 626.9 & $4.95 \mathrm{E}-03$ & 627.0 & $1.18 \mathrm{E}-02$ & 627.0 & $2.22 \mathrm{E}-02$ & $1,253.0$ & $2.31 \mathrm{E}-02$ & $1,253.0$ & $7.42 \mathrm{E}-03$ & 627.0 & $2.52 \mathrm{E}-02$ & 627.1 & $9.35 \mathrm{E}-03$ \\
\hline 7 & 754.4 & $2.71 \mathrm{E}-02$ & 754.4 & $5.04 \mathrm{E}-03$ & 754.2 & $1.20 \mathrm{E}-02$ & 754.4 & $2.25 \mathrm{E}-02$ & $1,502.4$ & $2.33 \mathrm{E}-02$ & $1,502.3$ & $7.47 \mathrm{E}-03$ & 754.4 & $2.68 \mathrm{E}-02$ & 754.5 & $9.77 \mathrm{E}-03$ \\
\hline 8 & 876.1 & $2.76 \mathrm{E}-02$ & 876.2 & $5.09 \mathrm{E}-03$ & 876.1 & $1.21 \mathrm{E}-02$ & 876.2 & $2.26 \mathrm{E}-02$ & $1,751.9$ & $2.35 \mathrm{E}-02$ & $1,751.8$ & $7.50 \mathrm{E}-03$ & 876.2 & $2.77 \mathrm{E}-02$ & 876.4 & $9.99 \mathrm{E}-03$ \\
\hline 9 & $1,003.4$ & 2.79E-02 & $1,003.4$ & $5.12 \mathrm{E}-03$ & $1,003.4$ & $1.22 \mathrm{E}-02$ & $1,003.5$ & $2.27 \mathrm{E}-02$ & $2,001.1$ & $2.36 \mathrm{E}-02$ & $2,001.1$ & $7.52 \mathrm{E}-03$ & $1,003.5$ & $2.83 \mathrm{E}-02$ & $1,003.7$ & $1.01 \mathrm{E}-02$ \\
\hline 10 & $1,125.7$ & $2.80 \mathrm{E}-02$ & $1,125.8$ & $5.14 \mathrm{E}-03$ & $1,125.7$ & $1.23 \mathrm{E}-02$ & $1,125.8$ & $2.28 \mathrm{E}-02$ & $2,255.5$ & $2.37 \mathrm{E}-02$ & $2,255.5$ & $7.54 \mathrm{E}-03$ & $1,125.9$ & $2.88 \mathrm{E}-02$ & $1,126.0$ & $1.02 \mathrm{E}-02$ \\
\hline 11 & $1,252.6$ & $2.82 \mathrm{E}-02$ & $1,252.7$ & $5.15 \mathrm{E}-03$ & $1,252.6$ & $1.23 \mathrm{E}-02$ & $1,252.7$ & $2.29 \mathrm{E}-02$ & $2,504.4$ & $2.37 \mathrm{E}-02$ & $2,504.2$ & $7.55 \mathrm{E}-03$ & $1,252.8$ & $2.91 \mathrm{E}-02$ & $1,253.0$ & $1.03 \mathrm{E}-02$ \\
\hline 12 & $1,374.9$ & $2.82 \mathrm{E}-02$ & $1,374.9$ & $5.16 \mathrm{E}-03$ & $1,374.9$ & $1.23 \mathrm{E}-02$ & $1,374.9$ & $2.29 \mathrm{E}-02$ & $2,753.8$ & $2.37 \mathrm{E}-02$ & $2,753.6$ & $7.55 \mathrm{E}-03$ & $1,375.0$ & $2.93 \mathrm{E}-02$ & $1,375.2$ & $1.03 \mathrm{E}-02$ \\
\hline 13 & $1,502.0$ & $2.83 \mathrm{E}-02$ & $1,502.1$ & $5.16 \mathrm{E}-03$ & $1,502.0$ & $1.24 \mathrm{E}-02$ & $1,502.1$ & $2.29 \mathrm{E}-02$ & $3,003.1$ & $2.38 \mathrm{E}-02$ & $3,002.9$ & $7.55 \mathrm{E}-03$ & $1,502.2$ & $2.94 \mathrm{E}-02$ & $1,502.4$ & $1.04 \mathrm{E}-02$ \\
\hline 14 & $1,629.1$ & $2.83 \mathrm{E}-02$ & $1,629.2$ & $5.17 \mathrm{E}-03$ & $1,629.2$ & $1.24 \mathrm{E}-02$ & $1,629.2$ & $2.30 \mathrm{E}-02$ & $3,252.3$ & $2.38 \mathrm{E}-02$ & $3,252.1$ & $7.55 \mathrm{E}-03$ & $1,629.4$ & $2.96 \mathrm{E}-02$ & $1,629.6$ & $1.04 \mathrm{E}-02$ \\
\hline 15 & $1,751.3$ & $2.84 \mathrm{E}-02$ & $1,751.5$ & $5.18 \mathrm{E}-03$ & $1,751.2$ & $1.24 \mathrm{E}-02$ & $1,751.4$ & $2.30 \mathrm{E}-02$ & $3,501.7$ & $2.38 \mathrm{E}-02$ & $3,501.4$ & $7.55 \mathrm{E}-03$ & $1,751.7$ & $2.96 \mathrm{E}-02$ & $1,751.8$ & $1.04 \mathrm{E}-02$ \\
\hline 16 & $1,878.3$ & $2.84 \mathrm{E}-02$ & $1,878.5$ & $5.18 \mathrm{E}-03$ & $1,878.4$ & $1.24 \mathrm{E}-02$ & $1,878.5$ & $2.30 \mathrm{E}-02$ & $3,755.6$ & $2.38 \mathrm{E}-02$ & $3,755.3$ & $7.56 \mathrm{E}-03$ & $1,878.6$ & 2.97E-02 & $1,878.8$ & $1.04 \mathrm{E}-02$ \\
\hline 17 & $2,000.5$ & $2.84 \mathrm{E}-02$ & $2,000.7$ & $5.18 \mathrm{E}-03$ & $2,000.6$ & $1.24 \mathrm{E}-02$ & $2,000.7$ & $2.30 \mathrm{E}-02$ & $4,004.7$ & $2.38 \mathrm{E}-02$ & $4,004.5$ & $7.56 \mathrm{E}-03$ & $2,000.9$ & $2.98 \mathrm{E}-02$ & $2,001.0$ & $1.05 \mathrm{E}-02$ \\
\hline 18 & $2,127.7$ & $2.84 \mathrm{E}-02$ & $2,128.0$ & $5.18 \mathrm{E}-03$ & $2,127.8$ & $1.24 \mathrm{E}-02$ & $2,127.9$ & $2.31 \mathrm{E}-02$ & $4,254.4$ & $2.38 \mathrm{E}-02$ & $4,254.2$ & $7.56 \mathrm{E}-03$ & $2,128.1$ & $2.98 \mathrm{E}-02$ & $2,128.3$ & $1.05 \mathrm{E}-02$ \\
\hline 19 & $2,254.9$ & $2.84 \mathrm{E}-02$ & $2,255.0$ & $5.19 \mathrm{E}-03$ & $2,254.9$ & $1.24 \mathrm{E}-02$ & $2,255.0$ & $2.31 \mathrm{E}-02$ & $4,503.4$ & $2.38 \mathrm{E}-02$ & $4,503.1$ & $7.56 \mathrm{E}-03$ & $2,255.2$ & $2.99 \mathrm{E}-02$ & $2,255.4$ & $1.05 \mathrm{E}-02$ \\
\hline 20 & $2,377.1$ & $2.85 \mathrm{E}-02$ & $2,377.3$ & $5.19 \mathrm{E}-03$ & $2,377.1$ & $1.24 \mathrm{E}-02$ & $2,377.2$ & $2.31 \mathrm{E}-02$ & $4,752.7$ & $2.38 \mathrm{E}-02$ & $4,752.4$ & $7.56 \mathrm{E}-03$ & $2,377.4$ & $2.99 \mathrm{E}-02$ & $2,377.7$ & $1.05 \mathrm{E}-02$ \\
\hline 21 & $2,503.6$ & $2.85 \mathrm{E}-02$ & $2,503.7$ & $5.19 \mathrm{E}-03$ & $2,503.6$ & $1.24 \mathrm{E}-02$ & $2,503.7$ & $2.31 \mathrm{E}-02$ & $5,001.6$ & $2.38 \mathrm{E}-02$ & $5,001.3$ & 7.57E-03 & $2,504.0$ & $2.99 \mathrm{E}-02$ & $2,504.2$ & $1.05 \mathrm{E}-02$ \\
\hline 22 & $2,626.0$ & 2.85E-02 & $2,626.2$ & $5.19 \mathrm{E}-03$ & $2,626.0$ & $1.24 \mathrm{E}-02$ & $2,626.2$ & $2.31 \mathrm{E}-02$ & $5,255.5$ & $2.38 \mathrm{E}-02$ & $5,255.2$ & 7.57E-03 & $2,626.3$ & $3.00 \mathrm{E}-02$ & $2,626.6$ & $1.05 \mathrm{E}-02$ \\
\hline 23 & $2,752.9$ & 2.85E-02 & $2,753.0$ & $5.20 \mathrm{E}-03$ & $2,752.8$ & $1.24 \mathrm{E}-02$ & $2,753.1$ & $2.31 \mathrm{E}-02$ & 5504.8 & $2.38 \mathrm{E}-02$ & $5,504.4$ & $7.57 \mathrm{E}-03$ & $2,753.2$ & $3.00 \mathrm{E}-02$ & $2,753.6$ & $1.05 \mathrm{E}-02$ \\
\hline 24 & $2,880.0$ & $2.85 \mathrm{E}-02$ & $2,880.2$ & $5.20 \mathrm{E}-03$ & $2,880.0$ & $1.25 \mathrm{E}-02$ & $2,880.2$ & $2.31 \mathrm{E}-02$ & $5,753.8$ & $2.38 \mathrm{E}-02$ & $5,753.4$ & 7.57E-03 & $2,880.4$ & $3.00 \mathrm{E}-02$ & $2,880.6$ & $1.05 \mathrm{E}-02$ \\
\hline 25 & $3,002.3$ & $2.85 \mathrm{E}-02$ & $3,002.3$ & $5.20 \mathrm{E}-03$ & $3,002.3$ & $1.25 \mathrm{E}-02$ & $3,002.5$ & $2.31 \mathrm{E}-02$ & $6,003.0$ & $2.38 \mathrm{E}-02$ & $6,002.6$ & 7.57E-03 & $3,002.7$ & $3.00 \mathrm{E}-02$ & $3,002.9$ & $1.05 \mathrm{E}-02$ \\
\hline 26 & $3,129.3$ & $2.85 \mathrm{E}-02$ & $3,129.4$ & $5.21 \mathrm{E}-03$ & $3,129.2$ & $1.25 \mathrm{E}-02$ & $3,129.5$ & $2.32 \mathrm{E}-02$ & $6,252.3$ & $2.38 \mathrm{E}-02$ & $6,251.9$ & $7.58 \mathrm{E}-03$ & $3,129.8$ & $3.00 \mathrm{E}-02$ & $3,130.0$ & $1.05 \mathrm{E}-02$ \\
\hline 27 & $3,251.4$ & $2.85 \mathrm{E}-02$ & $3,251.4$ & $5.21 \mathrm{E}-03$ & $3,251.3$ & $1.25 \mathrm{E}-02$ & $3,251.4$ & $2.32 \mathrm{E}-02$ & $6,506.3$ & $2.38 \mathrm{E}-02$ & $6,505.9$ & $7.57 \mathrm{E}-03$ & $3,251.7$ & $3.00 \mathrm{E}-02$ & $3,252.1$ & $1.05 \mathrm{E}-02$ \\
\hline 28 & $3,378.5$ & $2.85 \mathrm{E}-02$ & $3,378.5$ & $5.21 \mathrm{E}-03$ & $3,378.4$ & $1.25 \mathrm{E}-02$ & $3,378.5$ & $2.32 \mathrm{E}-02$ & $6,755.5$ & $2.39 \mathrm{E}-02$ & $6,755.0$ & $7.57 \mathrm{E}-03$ & $3,378.9$ & $3.01 \mathrm{E}-02$ & $3,379.2$ & $1.05 \mathrm{E}-02$ \\
\hline 29 & $3,500.6$ & $2.85 \mathrm{E}-02$ & $3,500.6$ & $5.21 \mathrm{E}-03$ & $3,500.5$ & $1.25 \mathrm{E}-02$ & $3,500.7$ & $2.32 \mathrm{E}-02$ & $7,004.3$ & $2.38 \mathrm{E}-02$ & $7,003.8$ & 7.57E-03 & $3,501.1$ & $3.01 \mathrm{E}-02$ & $3,501.4$ & $1.05 \mathrm{E}-02$ \\
\hline 30 & $3,627.6$ & $2.85 \mathrm{E}-02$ & $3,627.7$ & $5.21 \mathrm{E}-03$ & $3,627.6$ & $1.25 \mathrm{E}-02$ & $3,627.7$ & $2.32 \mathrm{E}-02$ & $7,253.6$ & $2.38 \mathrm{E}-02$ & $7,253.1$ & $7.58 \mathrm{E}-03$ & $3,628.1$ & $3.01 \mathrm{E}-02$ & $3,628.4$ & $1.05 \mathrm{E}-02$ \\
\hline 31 & $3,754.5$ & $2.85 \mathrm{E}-02$ & $3,754.5$ & $5.21 \mathrm{E}-03$ & $3,754.5$ & $1.25 \mathrm{E}-02$ & $3,754.6$ & $2.31 \mathrm{E}-02$ & $7,502.4$ & $2.38 \mathrm{E}-02$ & $7,501.9$ & 7.57E-03 & $3,754.9$ & $3.01 \mathrm{E}-02$ & $3,755.3$ & $1.05 \mathrm{E}-02$ \\
\hline 32 & $3,876.7$ & $2.85 \mathrm{E}-02$ & $3,876.7$ & $5.21 \mathrm{E}-03$ & $3,876.6$ & $1.25 \mathrm{E}-02$ & $3,876.8$ & $2.32 \mathrm{E}-02$ & $7,751.1$ & $2.39 \mathrm{E}-02$ & $7,750.6$ & 7.57E-03 & $3,877.2$ & $3.01 \mathrm{E}-02$ & $3,877.5$ & $1.05 \mathrm{E}-02$ \\
\hline 33 & $4,003.5$ & $2.85 \mathrm{E}-02$ & $4,003.7$ & $5.21 \mathrm{E}-03$ & $4,003.6$ & $1.25 \mathrm{E}-02$ & $4,003.7$ & $2.32 \mathrm{E}-02$ & $8,005.1$ & $2.38 \mathrm{E}-02$ & $8,004.5$ & $7.57 \mathrm{E}-03$ & $4,004.1$ & $3.01 \mathrm{E}-02$ & $4,004.5$ & $1.05 \mathrm{E}-02$ \\
\hline 34 & $4,126.3$ & $2.85 \mathrm{E}-02$ & $4,126.4$ & $5.21 \mathrm{E}-03$ & $4,126.3$ & $1.25 \mathrm{E}-02$ & $4,126.4$ & $2.32 \mathrm{E}-02$ & $8,254.1$ & $2.38 \mathrm{E}-02$ & $8,253.4$ & $7.58 \mathrm{E}-03$ & $4,126.9$ & $3.01 \mathrm{E}-02$ & $4,127.2$ & $1.05 \mathrm{E}-02$ \\
\hline 35 & $4,253.1$ & $2.85 \mathrm{E}-02$ & $4,253.3$ & $5.21 \mathrm{E}-03$ & $4,253.1$ & $1.25 \mathrm{E}-02$ & $4,253.3$ & $2.32 \mathrm{E}-02$ & $8,503.1$ & $2.39 \mathrm{E}-02$ & $8,502.5$ & $7.58 \mathrm{E}-03$ & $4,253.8$ & $3.01 \mathrm{E}-02$ & $4,254.1$ & $1.05 \mathrm{E}-02$ \\
\hline 36 & $4,380.0$ & $2.85 \mathrm{E}-02$ & $4,380.2$ & $5.21 \mathrm{E}-03$ & $4,380.1$ & $1.25 \mathrm{E}-02$ & $4,380.2$ & $2.32 \mathrm{E}-02$ & $8,751.9$ & $2.39 \mathrm{E}-02$ & $8,751.2$ & $7.57 \mathrm{E}-03$ & $4,380.7$ & $3.01 \mathrm{E}-02$ & $4,381.1$ & $1.05 \mathrm{E}-02$ \\
\hline 37 & $4,502.0$ & $2.85 \mathrm{E}-02$ & $4,502.2$ & $5.21 \mathrm{E}-03$ & $4,502.1$ & $1.25 \mathrm{E}-02$ & $4,502.3$ & $2.32 \mathrm{E}-02$ & $9,000.9$ & $2.39 \mathrm{E}-02$ & $9,000.2$ & $7.58 \mathrm{E}-03$ & $4,502.7$ & $3.01 \mathrm{E}-02$ & $4,503.1$ & $1.05 \mathrm{E}-02$ \\
\hline 38 & $4,629.1$ & $2.85 \mathrm{E}-02$ & $4,629.3$ & $5.21 \mathrm{E}-03$ & $4,629.1$ & $1.25 \mathrm{E}-02$ & $4,629.2$ & $2.32 \mathrm{E}-02$ & $9,250.0$ & $2.38 \mathrm{E}-02$ & $9,249.4$ & $7.58 \mathrm{E}-03$ & $4,629.7$ & $3.01 \mathrm{E}-02$ & $4,630.1$ & $1.05 \mathrm{E}-02$ \\
\hline 39 & $4,751.3$ & $2.85 \mathrm{E}-02$ & $4,751.4$ & $5.21 \mathrm{E}-03$ & $4,751.3$ & $1.25 \mathrm{E}-02$ & $4,751.5$ & $2.32 \mathrm{E}-02$ & $9,503.6$ & $2.39 \mathrm{E}-02$ & $9,503.0$ & $7.58 \mathrm{E}-03$ & $4,751.9$ & $3.01 \mathrm{E}-02$ & $4,752.4$ & $1.05 \mathrm{E}-02$ \\
\hline 40 & $4,878.3$ & $2.85 \mathrm{E}-02$ & $4,878.4$ & $5.21 \mathrm{E}-03$ & $4,878.3$ & $1.25 \mathrm{E}-02$ & $4,878.4$ & $2.32 \mathrm{E}-02$ & $9,752.8$ & $2.39 \mathrm{E}-02$ & $9,752.1$ & 7.57E-03 & $4,878.9$ & $3.01 \mathrm{E}-02$ & $4,879.4$ & $1.05 \mathrm{E}-02$ \\
\hline 41 & $5,004.8$ & $2.85 \mathrm{E}-02$ & $5,004.9$ & $5.22 \mathrm{E}-03$ & $5,004.8$ & $1.25 \mathrm{E}-02$ & $5,005.0$ & $2.32 \mathrm{E}-02$ & $10,001.6$ & $2.38 \mathrm{E}-02$ & $10,001.0$ & $7.58 \mathrm{E}-03$ & $5,005.5$ & $3.01 \mathrm{E}-02$ & $5,006.0$ & $1.05 \mathrm{E}-02$ \\
\hline
\end{tabular}

Notes: Field = applied field, $M=$ magnetization. Halo = samples from alteration halos, normal = samples from adjacent rock. 


\begin{tabular}{|c|c|c|c|c|c|c|c|c|c|c|c|c|c|c|c|c|}
\hline \multirow{3}{*}{$\begin{array}{l}\text { Core, section, } \\
\text { interval }(\mathrm{cm}) \text { : }\end{array}$} & \multicolumn{16}{|c|}{ Hole U1301B } \\
\hline & \multicolumn{2}{|c|}{$18 \mathrm{R}-1,36$} & \multicolumn{2}{|c|}{$18 \mathrm{R}-1,36$} & \multicolumn{2}{|c|}{$18 \mathrm{R}-4,140$} & \multicolumn{2}{|c|}{$18 \mathrm{R}-4,140$} & \multicolumn{2}{|c|}{$25 \mathrm{R}-2,79$} & \multicolumn{2}{|c|}{$25 \mathrm{R}-2,79$} & \multicolumn{2}{|c|}{$33 \mathrm{R}-2,138$} & \multicolumn{2}{|c|}{$33 \mathrm{R}-2,138$} \\
\hline & $\begin{array}{l}\text { Field } \\
\text { (Oe) }\end{array}$ & $\begin{array}{l}\text { Halo } M \\
\text { (emu) }\end{array}$ & $\begin{array}{l}\text { Field } \\
(\mathrm{Oe})\end{array}$ & $\begin{array}{l}\text { Normal } M \\
(\mathrm{emu})\end{array}$ & $\begin{array}{l}\text { Field } \\
\text { (Oe) }\end{array}$ & $\begin{array}{l}\text { Halo } M \\
\text { (emu) }\end{array}$ & $\begin{array}{l}\text { Field } \\
(\mathrm{Oe})\end{array}$ & $\begin{array}{l}\text { Normal } M \\
(\mathrm{emu})\end{array}$ & $\begin{array}{l}\text { Field } \\
(\mathrm{Oe})\end{array}$ & $\begin{array}{l}\text { Halo } M \\
\text { (emu) }\end{array}$ & $\begin{array}{l}\text { Field } \\
(\mathrm{Oe})\end{array}$ & $\begin{array}{l}\text { Normal } M \\
(\mathrm{emu})\end{array}$ & $\begin{array}{l}\text { Field } \\
(\mathrm{Oe})\end{array}$ & $\begin{array}{l}\text { Halo } M \\
\text { (emu) }\end{array}$ & $\begin{array}{l}\text { Field } \\
(\mathrm{Oe})\end{array}$ & $\begin{array}{l}\text { Normal } M \\
(\mathrm{emu})\end{array}$ \\
\hline 1 & 0.9 & $1.45 \mathrm{E}-04$ & $8.81 \mathrm{E}-01$ & 7.07E-05 & 0.8 & $1.83 \mathrm{E}-04$ & 0.8 & $2.92 \mathrm{E}-04$ & 0.2 & $1.58 \mathrm{E}-04$ & 0.9 & $1.99 \mathrm{E}-04$ & $8.45 \mathrm{E}-01$ & 1.49E-04 & $8.35 \mathrm{E}-01$ & 1.77E-04 \\
\hline 2 & 250.5 & $1.06 \mathrm{E}-02$ & $2.50 \mathrm{E}+02$ & 3.37E-03 & 128.0 & $1.60 \mathrm{E}-03$ & 128.0 & $3.44 \mathrm{E}-03$ & 50.1 & $3.29 \mathrm{E}-04$ & 128.0 & $5.56 \mathrm{E}-03$ & $2.50 \mathrm{E}+02$ & $9.80 \mathrm{E}-03$ & $2.50 \mathrm{E}+02$ & $1.08 \mathrm{E}-02$ \\
\hline 3 & 500.2 & $1.42 \mathrm{E}-02$ & $5.00 \mathrm{E}+02$ & $3.75 \mathrm{E}-03$ & 250.4 & $1.01 \mathrm{E}-02$ & 250.5 & $1.55 \mathrm{E}-02$ & 100.0 & $1.29 \mathrm{E}-03$ & 250.4 & $1.29 \mathrm{E}-02$ & $5.00 \mathrm{E}+02$ & $1.76 \mathrm{E}-02$ & $5.00 \mathrm{E}+02$ & $1.41 \mathrm{E}-02$ \\
\hline 4 & 754.5 & $1.50 \mathrm{E}-02$ & $7.54 \mathrm{E}+02$ & $3.84 \mathrm{E}-03$ & 377.6 & $1.85 \mathrm{E}-02$ & 377.6 & $2.36 \mathrm{E}-02$ & 150.0 & $4.13 \mathrm{E}-03$ & 377.5 & $1.53 \mathrm{E}-02$ & $7.54 \mathrm{E}+02$ & $1.97 \mathrm{E}-02$ & $7.54 \mathrm{E}+02$ & 1.46E-02 \\
\hline 5 & $1,003.8$ & $1.53 \mathrm{E}-02$ & $1.00 \mathrm{E}+03$ & 3.87E-03 & 500.0 & $2.29 \mathrm{E}-02$ & 500.0 & $2.69 \mathrm{E}-02$ & 199.9 & $8.21 \mathrm{E}-03$ & 500.0 & 1.60E-02 & $1.00 \mathrm{E}+03$ & $2.04 \mathrm{E}-02$ & $1.00 \mathrm{E}+03$ & 1.47E-02 \\
\hline 6 & $1,253.3$ & $1.54 \mathrm{E}-02$ & $1.25 \mathrm{E}+03$ & $3.88 \mathrm{E}-03$ & 626.9 & $2.53 \mathrm{E}-02$ & 626.9 & $2.85 \mathrm{E}-02$ & 251.2 & $1.24 \mathrm{E}-02$ & 626.9 & $1.62 \mathrm{E}-02$ & $1.25 \mathrm{E}+03$ & $2.07 \mathrm{E}-02$ & $1.25 \mathrm{E}+03$ & $1.48 \mathrm{E}-02$ \\
\hline 7 & $1,502.7$ & $1.55 \mathrm{E}-02$ & $1.50 \mathrm{E}+03$ & $3.89 \mathrm{E}-03$ & 754.3 & $2.66 \mathrm{E}-02$ & 754.4 & $2.93 \mathrm{E}-02$ & 301.1 & $1.58 \mathrm{E}-02$ & 754.3 & $1.63 \mathrm{E}-02$ & $1.50 \mathrm{E}+03$ & $2.09 \mathrm{E}-02$ & $1.50 \mathrm{E}+03$ & $1.48 \mathrm{E}-02$ \\
\hline 8 & $1,752.1$ & $1.55 \mathrm{E}-02$ & $1.75 \mathrm{E}+03$ & 3.90E-03 & 876.1 & $2.74 \mathrm{E}-02$ & 876.2 & $2.97 \mathrm{E}-02$ & 351.1 & $1.86 \mathrm{E}-02$ & 876.1 & $1.64 \mathrm{E}-02$ & $1.75 \mathrm{E}+03$ & $2.10 \mathrm{E}-02$ & $1.75 \mathrm{E}+03$ & $1.49 \mathrm{E}-02$ \\
\hline 9 & $2,001.4$ & $1.56 \mathrm{E}-02$ & $2.00 E+03$ & $3.90 \mathrm{E}-03$ & $1,003.4$ & $2.79 \mathrm{E}-02$ & $1,003.5$ & $3.00 \mathrm{E}-02$ & 400.9 & $2.07 \mathrm{E}-02$ & $1,003.4$ & $1.65 \mathrm{E}-02$ & $2.00 \mathrm{E}+03$ & $2.10 \mathrm{E}-02$ & $2.00 E+03$ & 1.49E-02 \\
\hline 10 & $2,255.8$ & $1.56 \mathrm{E}-02$ & $2.25 \mathrm{E}+03$ & $3.91 \mathrm{E}-03$ & $1,125.7$ & $2.82 \mathrm{E}-02$ & $1,125.8$ & 3.02E-02 & 450.8 & 2.23E-02 & $1,125.8$ & $1.65 \mathrm{E}-02$ & $2.26 \mathrm{E}+03$ & $2.11 \mathrm{E}-02$ & $2.26 \mathrm{E}+03$ & 1.49E-02 \\
\hline 11 & $2,504.7$ & $1.56 \mathrm{E}-02$ & $2.50 \mathrm{E}+03$ & $3.91 \mathrm{E}-03$ & $1,252.7$ & $2.84 \mathrm{E}-02$ & $1,252.7$ & $3.03 \mathrm{E}-02$ & 500.6 & $2.35 \mathrm{E}-02$ & $1,252.7$ & $1.65 \mathrm{E}-02$ & $2.50 \mathrm{E}+03$ & $2.11 \mathrm{E}-02$ & $2.50 \mathrm{E}+03$ & 1.49E-02 \\
\hline 12 & $2,754.2$ & $1.56 \mathrm{E}-02$ & $2.75 E+03$ & $3.92 \mathrm{E}-03$ & $1,374.8$ & $2.85 \mathrm{E}-02$ & $1,374.9$ & $3.04 \mathrm{E}-02$ & 550.5 & $2.44 \mathrm{E}-02$ & $1,374.9$ & $1.65 \mathrm{E}-02$ & $2.75 \mathrm{E}+03$ & $2.11 \mathrm{E}-02$ & $2.75 E+03$ & $1.49 \mathrm{E}-02$ \\
\hline 13 & $3,003.5$ & $1.56 \mathrm{E}-02$ & $3.00 \mathrm{E}+03$ & $3.92 \mathrm{E}-03$ & $1,501.9$ & $2.86 \mathrm{E}-02$ & $1,502.0$ & $3.05 \mathrm{E}-02$ & 600.5 & $2.51 \mathrm{E}-02$ & $1,502.0$ & $1.65 \mathrm{E}-02$ & $3.00 \mathrm{E}+03$ & $2.11 \mathrm{E}-02$ & $3.00 \mathrm{E}+03$ & 1.49E-02 \\
\hline 14 & $3,252.6$ & $1.56 \mathrm{E}-02$ & $3.25 \mathrm{E}+03$ & $3.92 \mathrm{E}-03$ & $1,629.1$ & $2.87 \mathrm{E}-02$ & $1,629.3$ & $3.05 \mathrm{E}-02$ & 651.9 & $2.56 \mathrm{E}-02$ & $1,629.2$ & $1.66 \mathrm{E}-02$ & $3.25 \mathrm{E}+03$ & $2.11 \mathrm{E}-02$ & $3.25 \mathrm{E}+03$ & 1.49E-02 \\
\hline 15 & $3,502.0$ & $1.56 \mathrm{E}-02$ & $3.50 \mathrm{E}+03$ & $3.92 \mathrm{E}-03$ & $1,751.3$ & $2.88 \mathrm{E}-02$ & $1,751.5$ & $3.06 \mathrm{E}-02$ & 701.8 & $2.61 \mathrm{E}-02$ & $1,751.5$ & $1.66 \mathrm{E}-02$ & $3.50 \mathrm{E}+03$ & $2.11 \mathrm{E}-02$ & $3.50 E+03$ & $1.49 \mathrm{E}-02$ \\
\hline 16 & $3,756.1$ & $1.56 \mathrm{E}-02$ & $3.75 E+03$ & $3.93 \mathrm{E}-03$ & $1,878.3$ & $2.88 \mathrm{E}-02$ & $1,878.6$ & $3.06 \mathrm{E}-02$ & 751.5 & $2.64 \mathrm{E}-02$ & $1,878.4$ & $1.66 \mathrm{E}-02$ & $3.76 \mathrm{E}+03$ & $2.11 \mathrm{E}-02$ & $3.75 E+03$ & 1.49E-02 \\
\hline 17 & $4,005.3$ & $1.56 \mathrm{E}-02$ & $4.00 \mathrm{E}+03$ & $3.93 \mathrm{E}-03$ & $2,000.5$ & $2.88 \mathrm{E}-02$ & $2,000.8$ & 3.07E-02 & 801.5 & $2.67 \mathrm{E}-02$ & $2,000.6$ & $1.66 \mathrm{E}-02$ & $4.00 \mathrm{E}+03$ & $2.11 \mathrm{E}-02$ & $4.00 \mathrm{E}+03$ & 1.49E-02 \\
\hline 18 & $4,254.9$ & $1.56 \mathrm{E}-02$ & $4.25 \mathrm{E}+03$ & $3.93 \mathrm{E}-03$ & $2,127.8$ & $2.89 \mathrm{E}-02$ & $2,128.0$ & $3.07 \mathrm{E}-02$ & 851.3 & $2.69 \mathrm{E}-02$ & $2,127.8$ & $1.66 \mathrm{E}-02$ & $4.25 \mathrm{E}+03$ & $2.11 \mathrm{E}-02$ & $4.25 \mathrm{E}+03$ & 1.49E-02 \\
\hline 19 & $4,503.9$ & $1.56 \mathrm{E}-02$ & $4.50 \mathrm{E}+03$ & $3.93 \mathrm{E}-03$ & $2,254.9$ & $2.89 \mathrm{E}-02$ & $2,255.0$ & $3.07 \mathrm{E}-02$ & 901.2 & $2.70 \mathrm{E}-02$ & $2,254.9$ & $1.66 \mathrm{E}-02$ & $4.50 \mathrm{E}+03$ & $2.11 \mathrm{E}-02$ & $4.50 \mathrm{E}+03$ & 1.49E-02 \\
\hline 20 & $4,753.5$ & $1.56 \mathrm{E}-02$ & $4.75 E+03$ & $3.93 \mathrm{E}-03$ & $2,377.1$ & $2.89 \mathrm{E}-02$ & $2,377.3$ & $3.07 \mathrm{E}-02$ & 951.2 & $2.72 \mathrm{E}-02$ & $2,377.1$ & $1.66 \mathrm{E}-02$ & $4.75 E+03$ & $2.12 \mathrm{E}-02$ & $4.75 E+03$ & 1.49E-02 \\
\hline 21 & $5,002.3$ & $1.56 \mathrm{E}-02$ & $5.00 \mathrm{E}+03$ & $3.93 \mathrm{E}-03$ & $2,503.6$ & $2.89 \mathrm{E}-02$ & $2,503.8$ & $3.08 \mathrm{E}-02$ & $1,001.0$ & $2.73 \mathrm{E}-02$ & $2,503.7$ & $1.66 \mathrm{E}-02$ & $5.00 \mathrm{E}+03$ & $2.12 \mathrm{E}-02$ & $5.00 \mathrm{E}+03$ & 1.49E-02 \\
\hline 22 & $5,256.1$ & $1.56 \mathrm{E}-02$ & $5.25 \mathrm{E}+03$ & $3.93 \mathrm{E}-03$ & $2,626.1$ & $2.90 \mathrm{E}-02$ & $2,626.2$ & $3.08 \mathrm{E}-02$ & $1,050.9$ & $2.74 \mathrm{E}-02$ & $2,626.1$ & $1.67 \mathrm{E}-02$ & $5.25 \mathrm{E}+03$ & $2.12 \mathrm{E}-02$ & $5.25 \mathrm{E}+03$ & 1.49E-02 \\
\hline 23 & $5,505.5$ & $1.56 \mathrm{E}-02$ & $5.50 \mathrm{E}+03$ & $3.93 \mathrm{E}-03$ & $2,752.9$ & $2.90 \mathrm{E}-02$ & $2,753.1$ & $3.08 \mathrm{E}-02$ & $1,102.2$ & $2.75 \mathrm{E}-02$ & $2,752.9$ & $1.67 \mathrm{E}-02$ & $5.50 \mathrm{E}+03$ & $2.12 \mathrm{E}-02$ & $5.50 \mathrm{E}+03$ & 1.49E-02 \\
\hline 24 & $5,754.4$ & $1.56 \mathrm{E}-02$ & $5.75 E+03$ & $3.92 \mathrm{E}-03$ & $2,880.0$ & $2.90 \mathrm{E}-02$ & $2,880.2$ & $3.08 \mathrm{E}-02$ & $1,152.1$ & $2.76 \mathrm{E}-02$ & $2,880.1$ & $1.67 \mathrm{E}-02$ & $5.75 \mathrm{E}+03$ & $2.12 \mathrm{E}-02$ & $5.75 E+03$ & 1.49E-02 \\
\hline 25 & $6,003.8$ & $1.56 \mathrm{E}-02$ & $6.00 E+03$ & $3.92 \mathrm{E}-03$ & $3,002.2$ & $2.90 \mathrm{E}-02$ & $3,002.4$ & $3.08 \mathrm{E}-02$ & $1,201.9$ & $2.76 \mathrm{E}-02$ & $3,002.3$ & $1.67 \mathrm{E}-02$ & $6.00 E+03$ & $2.12 \mathrm{E}-02$ & $6.00 E+03$ & 1.49E-02 \\
\hline 26 & $6,253.1$ & $1.56 \mathrm{E}-02$ & $6.25 \mathrm{E}+03$ & $3.92 \mathrm{E}-03$ & $3,129.2$ & $2.90 \mathrm{E}-02$ & $3,129.5$ & $3.08 \mathrm{E}-02$ & $1,251.9$ & $2.77 \mathrm{E}-02$ & $3,129.3$ & $1.67 \mathrm{E}-02$ & $6.25 \mathrm{E}+03$ & $2.12 \mathrm{E}-02$ & $6.25 \mathrm{E}+03$ & 1.49E-02 \\
\hline 27 & $6,507.0$ & $1.56 \mathrm{E}-02$ & $6.50 \mathrm{E}+03$ & $3.92 \mathrm{E}-03$ & $3,251.3$ & $2.90 \mathrm{E}-02$ & $3,251.6$ & $3.09 \mathrm{E}-02$ & $1,301.8$ & $2.77 \mathrm{E}-02$ & $3,251.4$ & $1.67 \mathrm{E}-02$ & $6.51 \mathrm{E}+03$ & $2.12 \mathrm{E}-02$ & $6.51 \mathrm{E}+03$ & 1.49E-02 \\
\hline 28 & $6,756.2$ & $1.56 \mathrm{E}-02$ & $6.75 E+03$ & $3.92 \mathrm{E}-03$ & $3,378.4$ & $2.90 \mathrm{E}-02$ & $3,378.6$ & $3.09 \mathrm{E}-02$ & $1,351.6$ & $2.78 \mathrm{E}-02$ & $3,378.5$ & $1.67 \mathrm{E}-02$ & $6.75 E+03$ & $2.12 \mathrm{E}-02$ & $6.75 E+03$ & 1.49E-02 \\
\hline 29 & $7,005.1$ & $1.56 \mathrm{E}-02$ & $7.00 \mathrm{E}+03$ & $3.92 \mathrm{E}-03$ & $3,500.5$ & $2.90 \mathrm{E}-02$ & $3,500.8$ & $3.09 \mathrm{E}-02$ & $1,401.5$ & $2.78 \mathrm{E}-02$ & $3,500.7$ & $1.67 \mathrm{E}-02$ & $7.00 \mathrm{E}+03$ & $2.12 \mathrm{E}-02$ & $7.00 \mathrm{E}+03$ & $1.49 \mathrm{E}-02$ \\
\hline 30 & $7,254.5$ & $1.56 \mathrm{E}-02$ & $7.25 \mathrm{E}+03$ & $3.92 \mathrm{E}-03$ & $3,627.5$ & $2.90 \mathrm{E}-02$ & $3,627.8$ & $3.09 \mathrm{E}-02$ & $1,451.4$ & $2.78 \mathrm{E}-02$ & $3,627.7$ & $1.67 \mathrm{E}-02$ & $7.25 \mathrm{E}+03$ & $2.11 \mathrm{E}-02$ & $7.25 \mathrm{E}+03$ & 1.49E-02 \\
\hline 31 & $7,503.3$ & $1.56 \mathrm{E}-02$ & $7.50 \mathrm{E}+03$ & $3.92 \mathrm{E}-03$ & $3,754.4$ & $2.90 \mathrm{E}-02$ & $3,754.7$ & $3.09 \mathrm{E}-02$ & $1,501.2$ & $2.79 \mathrm{E}-02$ & $3,754.6$ & $1.67 \mathrm{E}-02$ & $7.50 \mathrm{E}+03$ & $2.11 \mathrm{E}-02$ & $7.50 \mathrm{E}+03$ & 1.49E-02 \\
\hline 32 & $7,752.0$ & $1.56 \mathrm{E}-02$ & $7.75 E+03$ & $3.92 \mathrm{E}-03$ & $3,876.5$ & $2.90 \mathrm{E}-02$ & $3,876.9$ & $3.09 \mathrm{E}-02$ & $1,552.6$ & $2.79 \mathrm{E}-02$ & $3,876.7$ & $1.67 \mathrm{E}-02$ & $7.75 E+03$ & $2.11 \mathrm{E}-02$ & $7.75 E+03$ & 1.49E-02 \\
\hline 33 & $8,006.0$ & $1.56 \mathrm{E}-02$ & $8.00 \mathrm{E}+03$ & $3.92 \mathrm{E}-03$ & $4,003.5$ & $2.90 \mathrm{E}-02$ & $4,003.8$ & $3.09 \mathrm{E}-02$ & $1,602.4$ & 2.79E-02 & $4,003.7$ & $1.67 \mathrm{E}-02$ & $8.00 \mathrm{E}+03$ & $2.11 \mathrm{E}-02$ & $8.00 \mathrm{E}+03$ & 1.49E-02 \\
\hline 34 & $8,255.0$ & $1.56 \mathrm{E}-02$ & $8.25 \mathrm{E}+03$ & $3.92 \mathrm{E}-03$ & $4,126.2$ & $2.90 \mathrm{E}-02$ & $4,126.6$ & $3.09 \mathrm{E}-02$ & $1,652.3$ & $2.79 \mathrm{E}-02$ & $4,126.5$ & 1.67E-02 & $8.25 \mathrm{E}+03$ & $2.11 \mathrm{E}-02$ & $8.25 E+03$ & 1.49E-02 \\
\hline 35 & $8,504.0$ & $1.56 \mathrm{E}-02$ & $8.50 \mathrm{E}+03$ & $3.92 \mathrm{E}-03$ & $4,253.1$ & $2.90 \mathrm{E}-02$ & $4,253.5$ & $3.09 \mathrm{E}-02$ & $1,702.2$ & $2.79 \mathrm{E}-02$ & $4,253.3$ & 1.67E-02 & $8.50 \mathrm{E}+03$ & $2.11 \mathrm{E}-02$ & $8.50 E+03$ & 1.49E-02 \\
\hline 36 & $8,753.1$ & $1.56 \mathrm{E}-02$ & $8.75 \mathrm{E}+03$ & $3.92 \mathrm{E}-03$ & $4,380.0$ & $2.90 \mathrm{E}-02$ & $4,380.4$ & 3.09E-02 & $1,752.0$ & $2.80 \mathrm{E}-02$ & $4,380.2$ & $1.67 \mathrm{E}-02$ & $8.75 \mathrm{E}+03$ & $2.11 \mathrm{E}-02$ & $8.75 \mathrm{E}+03$ & 1.49E-02 \\
\hline 37 & $9,002.0$ & $1.56 \mathrm{E}-02$ & $9.00 \mathrm{E}+03$ & $3.91 \mathrm{E}-03$ & $4,502.0$ & $2.91 \mathrm{E}-02$ & $4,502.4$ & $3.09 \mathrm{E}-02$ & $1,801.9$ & $2.80 \mathrm{E}-02$ & $4,502.2$ & $1.67 \mathrm{E}-02$ & $9.00 \mathrm{E}+03$ & $2.11 \mathrm{E}-02$ & $9.00 \mathrm{E}+03$ & 1.49E-02 \\
\hline 38 & $9,251.1$ & $1.56 \mathrm{E}-02$ & $9.25 \mathrm{E}+03$ & $3.92 \mathrm{E}-03$ & $4,629.0$ & $2.91 \mathrm{E}-02$ & $4,629.4$ & $3.09 \mathrm{E}-02$ & $1,851.9$ & $2.80 \mathrm{E}-02$ & $4,629.3$ & $1.67 \mathrm{E}-02$ & $9.25 \mathrm{E}+03$ & $2.11 \mathrm{E}-02$ & $9.25 \mathrm{E}+03$ & 1.49E-02 \\
\hline 39 & $9,504.8$ & $1.56 \mathrm{E}-02$ & $9.50 \mathrm{E}+03$ & $3.92 \mathrm{E}-03$ & $4,751.2$ & $2.91 \mathrm{E}-02$ & $4,751.6$ & $3.09 \mathrm{E}-02$ & $1,901.7$ & $2.80 \mathrm{E}-02$ & $4,751.5$ & $1.67 \mathrm{E}-02$ & $9.50 \mathrm{E}+03$ & $2.11 \mathrm{E}-02$ & $9.50 \mathrm{E}+03$ & 1.49E-02 \\
\hline 40 & $9,754.0$ & $1.56 \mathrm{E}-02$ & $9.75 E+03$ & $3.92 \mathrm{E}-03$ & $4,878.3$ & $2.91 \mathrm{E}-02$ & $4,878.6$ & $3.09 \mathrm{E}-02$ & $1,953.0$ & $2.80 \mathrm{E}-02$ & $4,878.5$ & $1.67 \mathrm{E}-02$ & $9.75 E+03$ & $2.11 \mathrm{E}-02$ & $9.75 E+03$ & 1.49E-02 \\
\hline 41 & $10,002.9$ & $1.56 \mathrm{E}-02$ & $1.00 \mathrm{E}+04$ & $3.92 \mathrm{E}-03$ & $5,004.8$ & $2.91 \mathrm{E}-02$ & $5,005.1$ & $3.09 \mathrm{E}-02$ & $2,002.8$ & $2.80 \mathrm{E}-02$ & $5,005.0$ & 1.67E-02 & $1.00 \mathrm{E}+04$ & $2.11 \mathrm{E}-02$ & $1.00 \mathrm{E}+04$ & 1.49E-02 \\
\hline
\end{tabular}


Table T3. Basalt sample hysteresis parameters, Hole U1301B. (See table notes.)

\begin{tabular}{|c|c|c|c|c|c|c|c|c|c|}
\hline $\begin{array}{l}\text { Core, section, } \\
\text { interval }(\mathrm{cm})\end{array}$ & $\begin{array}{l}\text { Depth } \\
\text { (mbsf) }\end{array}$ & $M_{\mathrm{r}}$ & $M_{s}$ & $M_{\mathrm{r}} / M_{\mathrm{s}}$ & $H_{c}$ & $H_{\mathrm{cr}}$ & $H_{\mathrm{cr}} / H_{\mathrm{c}}$ & NRM & $T_{\mathrm{c}}$ \\
\hline \multicolumn{10}{|l|}{ 301-U1301B- } \\
\hline $2 \mathrm{R}-1,100$ & 358.10 & 3.103 & 10.500 & 0.294 & 110.50 & 151.80 & 1.374 & 4.055 & 339 \\
\hline $4 \mathrm{R}-1,51$ & 367.11 & 9.201 & 27.370 & 0.336 & 139.80 & 193.20 & 1.382 & 7.967 & 359 \\
\hline $5 \mathrm{R}-1,100$ & 377.30 & 5.270 & 17.220 & 0.306 & 90.53 & 128.40 & 1.418 & 4.358 & 349 \\
\hline $6 \mathrm{R}-2,48$ & 387.93 & 2.226 & 7.382 & 0.302 & 108.50 & 156.90 & 1.446 & 4.054 & 339 \\
\hline $12 \mathrm{R}-1,70$ & 429.60 & 1.911 & 11.570 & 0.165 & 42.15 & 90.47 & 2.146 & 15.500 & 128 \\
\hline $13 \mathrm{R}-1,45$ & 430.35 & 5.157 & 31.210 & 0.165 & 35.85 & 66.97 & 1.868 & 48.280 & 149 \\
\hline $13 \mathrm{R}-1,120$ & 431.10 & 2.360 & 8.820 & 0.215 & 64.20 & 94.83 & 1.477 & 0.434 & 179 \\
\hline $15 \mathrm{R}-2,70$ & 445.80 & 3.210 & 16.400 & 0.196 & 65.71 & 122.60 & 1.866 & 0.445 & 128 \\
\hline $15 \mathrm{R}-2,78$ & 445.88 & 2.693 & 12.800 & 0.210 & 67.43 & 137.80 & 2.044 & 27.130 & 159 \\
\hline $15 \mathrm{R}-2,127$ & 446.37 & 4.230 & 15.500 & 0.272 & 72.80 & 99.81 & 1.371 & 0.446 & 289 \\
\hline 15R-3, 24 & 446.84 & 2.500 & 12.620 & 0.198 & 67.86 & 108.20 & 1.594 & 42.100 & 219 \\
\hline $15 \mathrm{R}-3,120$ & 447.80 & 2.758 & 12.240 & 0.225 & 59.14 & 90.59 & 1.532 & 41.453 & 260 \\
\hline $15 R-4,30$ & 448.4 & 4.307 & 20.480 & 0.210 & 53.99 & 82.78 & 1.533 & 16.771 & 370 \\
\hline $15 R-4,115$ & 449.25 & 3.022 & 13.370 & 0.226 & 65.18 & 121.30 & 1.861 & 58.429 & 190 \\
\hline $18 \mathrm{R}-1,55$ & 471.55 & 4.071 & 12.570 & 0.324 & 103.60 & 150.40 & 1.452 & 6.583 & 339 \\
\hline $18 \mathrm{R}-2,50$ & 472.80 & 1.782 & 15.280 & 0.117 & 36.42 & 81.63 & 2.241 & 19.308 & 129 \\
\hline $18 \mathrm{R}-2,102$ & 473.32 & 1.370 & 13.400 & 0.102 & 35.09 & 74.00 & 2.109 & 12.552 & 149 \\
\hline 18R-3, 25 & 473.81 & 1.638 & 13.510 & 0.121 & 35.33 & 74.40 & 2.106 & 35.066 & 129 \\
\hline $18 \mathrm{R}-3,58$ & 474.14 & 1.046 & 15.260 & 0.069 & 43.97 & 61.69 & 1.403 & 23.534 & 128 \\
\hline $18 R-4,112$ & 476.00 & 2.930 & 6.656 & 0.441 & 190.40 & 257.10 & 1.350 & 1.522 & 339 \\
\hline $21 \mathrm{R}-2,30$ & 491.86 & 2.890 & 6.478 & 0.446 & 159.30 & 207.50 & 1.303 & 2.774 & 369 \\
\hline $21 \mathrm{R}-2,138$ & 492.94 & 3.007 & 9.997 & 0.301 & 107.60 & 153.90 & 1.430 & 2.047 & 349 \\
\hline 23R-1, 28 & 500.18 & 3.275 & 10.390 & 0.315 & 108.30 & 159.70 & 1.475 & 8.647 & 339 \\
\hline $23 \mathrm{R}-2,30$ & 501.66 & 4.630 & 14.560 & 0.317 & 125.30 & 173.00 & 1.381 & 2.847 & 334 \\
\hline $23 \mathrm{R}-2,89$ & 502.25 & 6.003 & 15.620 & 0.384 & 166.80 & 215.90 & 1.294 & 3.686 & 349 \\
\hline $24 \mathrm{R}-1,10$ & 506.00 & 4.949 & 10.090 & 0.491 & 203.60 & 259.30 & 1.274 & 2.941 & 359 \\
\hline $24 \mathrm{R}-1,55$ & 506.45 & 2.302 & 5.155 & 0.447 & 170.70 & 222.70 & 1.305 & 3.601 & 359 \\
\hline $34 \mathrm{R}-2,90$ & 563.00 & 3.086 & 14.100 & 0.219 & 71.02 & 113.20 & 1.594 & 10.519 & 349 \\
\hline $35 \mathrm{R}-1,72$ & 564.32 & 4.518 & 12.540 & 0.360 & 129.10 & 178.90 & 1.386 & 1.507 & 365 \\
\hline $35 R-2,96$ & 566.03 & 3.290 & 11.000 & 0.325 & 110.00 & 158.00 & 1.440 & 1.507 & 364 \\
\hline $35 \mathrm{R}-2,130$ & 566.37 & 3.285 & 10.110 & 0.325 & 110.00 & 158.90 & 1.445 & 4.829 & 359 \\
\hline $36 \mathrm{R}-1,12$ & 573.32 & 3.977 & 9.824 & 0.405 & 162.50 & 221.60 & 1.364 & 2.602 & 369 \\
\hline
\end{tabular}

Notes: $M_{\mathrm{r}}=$ saturation remanence, $M_{\mathrm{s}}=$ saturation magnetization. $H_{\mathrm{c}}=$ remanent coercive force, $H_{\mathrm{cr}}=$ ordinary coercive force. NRM = natural remanent magnetization. $T_{\mathrm{c}}=$ Curie temperature.

Table T4. Hysteresis measurements from halo/nonhalo paired samples, Hole U1301B. (See table notes.)

\begin{tabular}{|c|c|c|c|c|c|c|c|c|}
\hline $\begin{array}{l}\text { Core, section, } \\
\text { interval }(\mathrm{cm})\end{array}$ & Type & $\begin{array}{c}M_{\mathrm{s}} \\
(\mathrm{emu})\end{array}$ & $\begin{array}{c}M_{\mathrm{r}} \\
(\mathrm{emu})\end{array}$ & $\begin{array}{c}H_{\mathrm{c}} \\
(\mathrm{Oe})\end{array}$ & $S^{*}$ & $\begin{array}{c}H_{\mathrm{cr}} \\
(\mathrm{Oe})\end{array}$ & $H_{\mathrm{cr}} / H_{\mathrm{c}}$ & $M_{\mathrm{rs}} / M_{\mathrm{s}}$ \\
\hline \multicolumn{9}{|l|}{ 301-U1301B- } \\
\hline $7 \mathrm{R}-1,12$ & Halo & 0.0654 & 0.0290 & 177.90 & 0.27 & 230.80 & 1.30 & 0.44 \\
\hline $7 \mathrm{R}-1,12$ & Normal & 0.0161 & 0.0053 & 112.70 & 0.18 & 189.90 & 1.69 & 0.33 \\
\hline $15 \mathrm{R}-1,44$ & Halo & 0.0419 & 0.0125 & 87.28 & 0.12 & 119.80 & 1.37 & 0.30 \\
\hline $15 \mathrm{R}-1,44$ & Normal & 0.1003 & 0.0232 & 60.60 & 0.09 & 96.30 & 1.59 & 0.23 \\
\hline $15 \mathrm{R}-2,141$ & Halo & 0.0646 & 0.0241 & 150.80 & 0.17 & 208.00 & 1.38 & 0.37 \\
\hline $15 \mathrm{R}-2,141$ & Normal & 0.0206 & 0.0077 & 135.90 & 0.16 & 250.00 & 1.84 & 0.37 \\
\hline $15 R-4,135$ & Halo & 0.0596 & 0.0305 & 206.30 & 0.21 & 255.40 & 1.24 & 0.51 \\
\hline $15 R-4,135$ & Normal & 0.0213 & 0.0106 & 180.70 & 0.25 & 227.80 & 1.26 & 0.50 \\
\hline 18R-1, 36 & Halo & 0.0435 & 0.0157 & 105.40 & 0.19 & 157.70 & 1.50 & 0.36 \\
\hline $18 \mathrm{R}-1,36$ & Normal & 0.0223 & 0.0039 & 42.21 & 0.10 & 135.40 & 3.21 & 0.18 \\
\hline $18 \mathrm{R}-4,140$ & Halo & 0.0581 & 0.0296 & 200.80 & 0.27 & 244.90 & 1.22 & 0.51 \\
\hline $18 \mathrm{R}-4,140$ & Normal & 0.0727 & 0.0313 & 147.20 & 0.26 & 201.30 & 1.37 & 0.43 \\
\hline $25 \mathrm{R}-2,79$ & Halo & 0.0706 & 0.0287 & 160.70 & 0.22 & 211.80 & 1.32 & 0.41 \\
\hline $25 \mathrm{R}-2,79$ & Normal & 0.0542 & 0.0168 & 84.62 & 0.20 & 121.50 & 1.44 & 0.31 \\
\hline $33 \mathrm{R}-2,138$ & Halo & 0.0584 & 0.0214 & 148.40 & 0.21 & 199.50 & 1.34 & 0.37 \\
\hline $33 \mathrm{R}-2,138$ & Normal & 0.0506 & 0.0150 & 86.90 & 0.17 & 150.90 & 1.74 & 0.30 \\
\hline
\end{tabular}

Notes: $M_{\mathrm{s}}=$ saturation magnetization, $M_{\mathrm{r}}=$ saturation remanence. $H_{\mathrm{c}}=$ remanent coercive force, $H_{\mathrm{cr}}=$ ordinary coercive force. $S^{*}=2\left(H 2 / H_{\mathrm{c}}\right)-1$, where $H 2$ is field value corresponding to $1 / 2 M_{r}$. 